\title{
Quantum Criticality via Magnetic Branes
}

\author{
Eric D’Hoker and Per Kraus
}

\begin{abstract}
Holographic methods are used to investigate the low temperature limit, including quantum critical behavior, of strongly coupled 4-dimensional gauge theories in the presence of an external magnetic field, and finite charge density. In addition to the metric, the dual gravity theory contains a Maxwell field with Chern-Simons coupling. In the absence of charge, the magnetic field induces an RG flow to an infrared $\mathrm{AdS}_{3} \times \mathbf{R}^{2}$ geometry, which is dual to a 2-dimensional CFT representing strongly interacting fermions in the lowest Landau level. Two asymptotic Virasoro algebras and one chiral Kac-Moody algebra arise as emergent symmetries in the IR. Including a nonzero charge density reveals a quantum critical point when the magnetic field reaches a critical value whose scale is set by the charge density. The critical theory is probed by the study of long-distance correlation functions of the boundary stress tensor and current. All quantities of major physical interest in this system, such as critical exponents and scaling functions, can be computed analytically. We also study an asymptotically $\mathrm{AdS}_{6}$ system whose magnetic field induced quantum critical point is governed by a IR Lifshitz geometry, holographically dual to a $\mathrm{D}=2+1$ field theory. The behavior of these holographic theories shares important similarities with that of real world quantum critical systems obtained by tuning a magnetic field, and may be relevant to materials such as Strontium Ruthenates.
\end{abstract}

Eric D'Hoker

Department of Physics and Astronomy, University of California, Los Angeles, CA 90095, USA, e-mail: dhokerephysics.ucla.edu

Per Kraus

Department of Physics and Astronomy, University of California, Los Angeles, CA 90095, USA, e-mail: pkraus@physics.ucla.edu 


\section{Introduction}

A statistical mechanics system undergoes a quantum phase transition when its ground state suffers a macroscopic rearrangement as an external parameter is varied. While a quantum phase transition takes place strictly at zero temperature, its presence governs quantum critical behavior in a small region of low temperature surrounding the quantum critical point. The existence of such a quantum critical region is believed to influence physics also at intermediate temperatures, and to have relevance to the phase structure of high- $T_{c}$ superconductors and strange metals [58].

Holography provides concrete tools for studying non-Abelian gauge dynamics in terms of classical solutions to Einstein's equations of gravity, provided the number of colors $N$ and the 't Hooft coupling $\lambda=N g_{\mathrm{YM}}^{2}$ are both large [50, 34, 62]. The classic example of this gauge/gravity duality relates $\mathscr{N}=4$ super Yang-Mills theory in 3+1 dimensional Minkowski space-time to Type IIB supergravity on $A d S_{5} \times S^{5}$. Global symmetries match under the duality: the space-time isometry group $S O(4,2) \times S O(6)$ on the gravity side maps to the conformal group $S O(4,2)$ and the R-symmetry group $S U(4) \sim S O(6)$ on the gauge theory side, while the number of supersymmetries is the maximal allowed 32 on both sides (for general reviews on the AdS/CFT correspondence, see for example [2, 46, 15, 55]).

The gauge/gravity duality continues to apply to systems with fewer or no supersymmetries, and with broken conformal and Poincaré invariance. For example, renormalization group flow away from a conformal invariant gauge theory is dual to a space-time which is asymptotically $A d S_{5}$ but deviates from $A d S_{5}$ away from the asymptotic limit. The dual to a gauge theory at finite temperature $T$ is a space-time containing a black hole or black brane whose Hawking temperature is $T$. A charge density or chemical potential, and a background magnetic field may all be incorporated in the gauge theory as well via precise dual gravity prescriptions, thereby setting the stage for applying holographic methods to a wide variety of interesting strongly coupled systems in statistical mechanics. Reviews on the applications of holographic methods to condensed matter problems may be found in [35, 40].

Whether holography will ever be able to model reliably the condensed state of a specific compound remains to be seen. What has become clear, however, is that gauge/gravity duality can provide quantitative information on universal behavior, such as critical phenomena, critical exponents, transport properties, and the like for certain classes of strongly coupled systems. One of the first examples derived in this spirit is the ratio of the shear viscosity to the entropy density [56]; a more recent one gives a bound on the specific heat exponent [61].

In the present paper, we shall review recent holographic investigations into the critical behavior of gauge theory at finite temperature $T$, with electric charge density $\rho$, and subjected to an external magnetic field $B$. Supersymmetry will play no significant role here, as the gauge theory in question may be supersymmetric or not. We shall be interested in the thermodynamic properties of this system especially at low temperatures, as well as in the behavior of correlators at long distances. 
Fig. 1 The holographic picture dual to a meta-magnetic quantum phase transition is given by the gradual expulsion of electric charge from the region interior to the event horizon of a black brane to the region outside the horizon. The quantum critical point corresponds to the transition point at which all electric charge resides outside the horizon of the black brane.

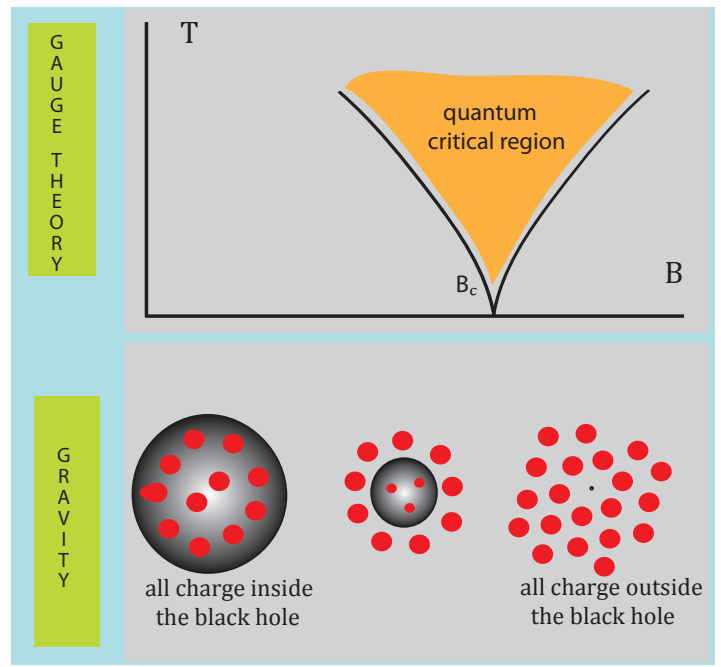

The holographic dual to this system, in the large $N$ and large $\lambda$ approximations, is provided by a theory of gravity in $4+1$ space-time dimensions, plus an Abelian gauge field. Anomalies in the gauge current of the 3+1-dimensional field theory side force the presence of a Chern-Simons term in the 4+1-dimensional theory. The CS coupling is the only dimensionless free parameter in the theory, and the holographic dynamics will depend crucially on its value. All our results will be derived using this holographic model [16, 17].

Using holographic methods, a rich structure is found which exhibits quantum critical behavior [18, 19, 21], and the emergence of a 1+1-dimensional CFT in IR correlators [22, 20]. Specifically, the system exhibits a quantum phase transition as the magnetic field $B$ crosses a critical value $B_{c}$, the scale for which is set by the charge density $\rho$ by $B_{c} \sim \rho^{2 / 3}$. Quantum critical behavior governs a region, depicted schematically in figure 1, where temperature is the largest scale. The mechanism underlying this transition on the gravity side is also illustrated in figure 1 it is driven by the expulsion of electric charge from within the horizon to the outside. More specifically, for vanishing magnetic field, the gravity solution is the AdS ReissnerNordstrom black brane which has non-zero charge density and entropy density at $T=0$. As $B$ is increased electric charge gets expelled from within the black brane horizon to the outside, up till $B=B_{c}$ at which value the black brane carries no more charge or entropy density. This charge expulsion mechanism is realized in other holographic systems as well [37]. Other examples of magnetic field driven holographic phase transitions include [47, 43]. 


\section{Basic Gauge Theory Dynamics}

Before embarking on the study of strongly coupled gauge theory with the tools of holography, we shall summarize here some basic results on the dynamics of gauge theory in the presence of an external magnetic field.

\subsection{Effective low energy degrees of freedom}

In the presence of a constant magnetic field $B$, the energy levels of massless free bosons and fermions of electric charge $q$ are given as follows,

$$
\begin{array}{lll}
\text { bosons } & E=\sqrt{p^{2}+(2 n+1)|q B|} & n=0,1,2, \cdots \\
\text { fermions } & E=\sqrt{p^{2}+2 n|q B|} & n=0,1,2, \cdots
\end{array}
$$

Here $p$ is the momentum component parallel to the magnetic field $B$. The energy levels for bosons and fermions clearly do not match, so that supersymmetry is manifestly broken. Simple modifications in which supersymmetry is restored do exist, however, and were studied in [5].

For large $B$, only fermions in the lowest Landau level remain massless. More precisely, the fermions in the lowest Landau level are 1+1-dimensional Weyl fermions, moving along the direction of the magnetic field, with momentum $p$, their chirality being correlated with their charge,

$$
\begin{array}{lll}
B q>0 & p>0 & \text { field of right-movers } \psi_{R} \\
B q<0 & p<0 & \text { field of left-movers } \psi_{L}
\end{array}
$$

All higher fermion levels and all boson levels acquire a large effective mass and will decouple from the spectrum.

In $\mathscr{N}=4$ super-Yang-Mills theory, the operator of charges $Q$ to which the magnetic field couples takes values in the R-symmetry algebra $S U(4)_{R}$. Although the gauge fields $A_{\mu}$, gauginos $\lambda_{\alpha}$, and scalars $\phi$ are now all strongly interacting, the above decoupling of charged fields will persist. Thus, the low energy effective degrees of freedom will be Weyl fermions $\psi_{L}, \psi_{R}$, whose coupling is induced by the remaining neutral gauge dynamics of the $A_{\mu}$ fields, and any remaining components of $\lambda$ and $\phi$ which are neutral under $Q$. This system of Weyl fermions should underlie an interacting conformal field theory in $1+1$ dimensions. Any asymmetry in the spectrum of charges $Q$ will give rise to the chiral magnetic effect, the observation of which is being considered in heavy ion collision experiments at RHIC [45].

At finite density and low or zero temperature, fermionic levels will fill up to a certain Fermi energy $E_{F}$. For the effective 1+1-dimensional CFT discussed here, the Fermi surface consists of just two points corresponding to two values of the Fermi 
momentum. The left- and right-movers $\psi_{L}$ and $\psi_{R}$ live separately at $k=k_{F, L}$ and $k=k_{F, R}$ respectively. If the spectrum of charges is symmetric then $k_{F, L}=-k_{F, R}$, as is illustrated in figure 2] But in general, $k_{F, L} \neq-k_{F, R}$, and the ground state of the system will carry a nonzero total momentum. As the charge density $\rho$ is being increased, $E_{F}$ will increase. When $E_{F}$ reaches the next Landau level, or the energies characteristic of the fully 3-dimensional excitations, a large number of degrees of freedom are being excited, and we may expect a quantum phase transition, at a critical charge density $\rho_{c}$, whose scale is set by the only possible dimensional scale in the problem, namely $\rho_{c} \sim B^{3 / 2}$.

Fig. 2 At finite charge density, fermion levels fill up to a Fermi energy $E_{F}$, corresponding to Fermi momenta $k_{F, L}$ and $k_{F, R}$. The low temperature degrees of freedom are chiral fermions $\psi_{L}$ and $\psi_{R}$ which reside at the Fermi "surface", consisting here of only two points $k=k_{F, L}$ and $k=k_{F, R}$. In the charge symmetric case we have $k_{F, L}=-k_{F, R}$.

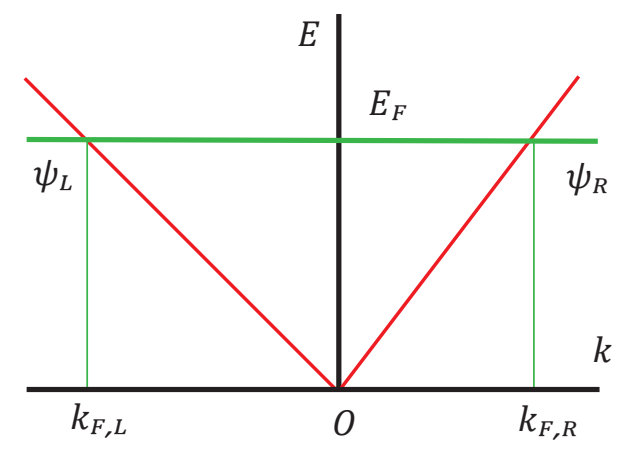

\subsection{Luttinger liquids}

The standard quantum field theory approach to systems of 1+1-dimensional interacting chiral fermions is provided by the Luttinger approach to quantum liquids. One begins by identifying the excitations near the Fermi surface, in this case the Weyl fermions $\psi_{L}, \psi_{R}$ introduced above. The Hamiltonian consists of bilinear terms which result from linearization around the Fermi surface, as well as all possible local four-fermi interactions compatible with the symmetries of the system,

$$
H_{\text {int }}=g_{2}\left(\psi_{L}^{\dagger} \psi_{L}\right)\left(\psi_{R}^{\dagger} \psi_{R}\right)+\frac{g_{4}}{2}\left(\psi_{L}^{\dagger} \psi_{L}\right)^{2}+\frac{g_{4}}{2}\left(\psi_{R}^{\dagger} \psi_{R}\right)^{2}
$$

Although the system was first solved in terms of fermionic fields by Dzyaloshinski and Larkin, modern methods based on bosonization provide a powerful reformulation in terms of two non-interacting boson fields [32]. A key thermodynamic relation for the entropy density $s_{\text {gauge }}$ as a function of the temperature $T$ is given by,

$$
s_{\text {gauge }}=\frac{\pi}{3 v} T \quad v=v_{F} \sqrt{\left(1+\frac{g_{4}}{2 \pi v_{F}}\right)^{2}-\left(\frac{g_{2}}{2 \pi v_{F}}\right)^{2}}
$$


where $v_{F}$ is the Fermi velocity, and $v$ the actual velocity of the chiral excitations. Correlators may be obtained as well. For example, the two-point function of the charge density $\rho(x)$ takes the following form,

$$
\langle\rho(x) \rho(0)\rangle=\frac{c_{0}}{x^{2}}+c_{\Delta} \frac{\cos \left(2 k_{F} x\right)}{x^{2 \Delta}}+\cdots
$$

where $c_{0}$ and $c_{\Delta}$ are constants, and $\Delta$ is the scaling dimension of the lowest dimensional operator which exchanges charge between $\psi_{L}$ and $\psi_{R}$.

\section{Holographic Dual set-up}

In this section, we shall discuss the basic set up for the holographic dual in the supergravity approximation. Since we shall concentrate on thermodynamics, as well as on correlators of energy density, momentum density, and charge density, we may limit the quantum field operators to the stress tensor $\mathscr{T}^{\mu \nu}$ and the Maxwell current $\mathscr{J}^{\mu}$ of the 3+1-dimensional gauge theory. The holographic dual fields to these operators are respectively the metric $g_{M N}$ and the Maxwell field $A_{M}$ of the 4+1-dimensional Einstein-Maxwell-Chern-Simons theory with action 1

$$
S=-\frac{1}{16 \pi G_{5}} \int d^{5} x \sqrt{g}\left(R-\frac{12}{\ell^{2}}+F_{M N} F^{M N}\right)+\frac{k}{12 \pi G_{5}} \int A \wedge F \wedge F
$$

Boundary as well as counterterm contributions to the action have not been exhibited here. Furthermore, $G_{5}$ is Newton's constant in $4+1$ dimensions, $-12 / \ell^{2}$ is the cosmological constant, and $k$ is the dimensionless Chern-Simons coupling. The anomaly of the chiral current $\mathscr{J}^{\mu}$ in the gauge theory is proportional to $k$, and we have $\partial_{\mu} \mathscr{J}^{\mu} \propto k \mathbf{E} \cdot \mathbf{B}$. The action is invariant under simultaneous reversal of the sign of $A$ and $k$, allowing us to restrict attention to $k \geq 0$, without loss of generality.

For the special value $k=k_{\text {susy }}=2 / \sqrt{3}$, the action $S$ coincides with the bosonic part of minimal supergravity in $4+1$ dimensions, and as such corresponds to a consistent truncation of all supersymmetric asymptotically $A d S_{5}$ compactifications of either Type IIB supergravity or M-theory [31]. Here, however, we shall leave $k$ a free parameter, and investigate the phase diagram as a function of $k$.

\subsection{Field equations and structure of the solutions}

The Bianchi identity is $d F=0$, while the field equations are given as follows,

\footnotetext{
${ }^{1}$ Einstein indices $\mu, v=0,1,2,3$ will be used in 3+1-dimensions, while Einstein indices $M, N=$ $0,1,2,3,4$ will be used in $4+1$-dimensions. Our conventions are $g=-\operatorname{det}\left(g_{M N}\right)$, as well as $R_{M N K}^{L}=\partial_{K} \Gamma_{M N}^{L}-\partial_{N} \Gamma_{M K}^{L}+\Gamma_{M N}^{P} \Gamma_{K P}^{L}-\Gamma_{M K}^{P} \Gamma_{N P}^{L}$ with $R_{M N}=R^{L}{ }_{M L N}$ and $R=g^{M N} R_{M N}$.
} 


$$
\begin{aligned}
0 & =d * F+k F \wedge F \\
R_{M N} & =\frac{4}{\ell^{2}} g_{M N}+\frac{1}{3} g_{M N} F^{P Q} F_{P Q}-2 F_{M P} F_{N}{ }^{P}
\end{aligned}
$$

For vanishing Maxwell field $F=0$, the field equations admit the $A d S_{5}$ solution of radius $\ell$. Henceforth, we shall set $\ell=1$. Denoting the coordinates of 4 -dimensional space-time by $x^{\mu}=\left(t, x_{1}, x_{2}, x_{3}\right)$, and the holographic coordinate by $r$, the $A d S_{5}$ solution takes the form,

$$
d s^{2}=g_{M N} d x^{M} d x^{N}=\frac{d r^{2}}{4 r^{2}}+2 r\left(-d t^{2}+d x_{1}^{2}+d x_{2}^{2}+d x_{3}^{2}\right)
$$

Introducing a constant uniform magnetic field $B$ along the direction $x_{3}$, and anticipating also the inclusion of finite temperature $T$, and constant uniform charge density $\rho$, we see that the symmetries to be imposed on the solutions should include,

1. Translation invariance in the coordinates $t, x_{1}, x_{2}, x_{3}$;

2. Rotation invariance in the $x_{1}, x_{2}$-plane.

The most general Ansatz consistent with these requirements is given by,

$$
\begin{aligned}
F & =B d x_{1} \wedge d x_{2}+E d r \wedge d t-P d r \wedge d x_{3}+\tilde{P} d t \wedge d x_{3} \\
d s^{2} & =f^{-1} d r^{2}+M d t^{2}+2 L d t d x_{3}+N d x_{3}^{2}+K\left(d x_{1}^{2}+d x_{2}^{2}\right)
\end{aligned}
$$

where all coefficient functions $B, E, P, \tilde{P}, f, K, L, M, N$ depend only on $r$. In view of the Bianchi identities, $B$ and $\tilde{P}$ must be independent of $r$, and in view of the field equations, we have $\tilde{P}=0$. This constant $B$ is nothing but the constant magnetic background field. Finally, the residual reparametrization invariance in the variable $r$ allows us to choose a coordinate $r$ such that

$$
f=L^{2}-M N
$$

a choice which will prove convenient throughout.

\subsection{Boundary stress tensor and current}

We will be considering asymptotically $\mathrm{AdS}_{5}$ solutions, for which the metric and gauge field admit a Fefferman-Graham expansion [29]. Introducing a radial coordinate $\rho$, defined such that the $\operatorname{AdS}_{5}$ boundary is located at $\rho=\infty$, the FeffermanGraham gauge choice puts the fields in the following form,

$$
\begin{aligned}
A & =A_{\mu}(\rho, x) d x^{\mu} \\
d s^{2} & =\frac{d \rho^{2}}{4 \rho^{2}}+g_{\mu v}(\rho, x) d x^{\mu} d x^{v}
\end{aligned}
$$

and their expansion in large $\rho$ takes the following form, 


$$
\begin{aligned}
A_{\mu}(\rho, x) & =A_{\mu}^{(0)}(x)+\frac{1}{\rho} A_{\mu}^{(2)}(x)+\cdots \\
g_{\mu \nu}(\rho, x) & =\rho g_{\mu \nu}^{(0)}(x)+g_{\mu \nu}^{(2)}(x)+\frac{1}{\rho} g_{\mu \nu}^{(4)}(x)+\frac{\ln \rho}{\rho} g_{\mu \nu}^{(\ln )}(x)+\cdots
\end{aligned}
$$

The coefficients $g_{\mu \nu}^{(4)}, g_{\mu \nu}^{(\mathrm{ln})}$, and the trace of $g_{\mu \nu}^{(4)}$ are fixed by the Einstein equations to be local functionals of the conformal boundary metric $g_{\mu \nu}^{(0)}$. The boundary stress tensor $T^{\mu v}$ and current $J^{\mu}$ of [9, 14] are defined in terms of the variation of the on-shell action with respect to $g_{\mu \nu}^{(0)}$ and $A_{\mu}^{(0)}$ respectively (see [22]). In terms of the Fefferman-Graham data the result is,

$$
\begin{aligned}
4 \pi G_{5} T_{\mu v}(x) & =g_{\mu \nu}^{(4)}(x)+\text { local } \\
2 \pi G_{5} J_{\mu}(x) & =A_{\mu}^{(2)}(x)+\text { local }
\end{aligned}
$$

Indices are raised and lowered using the conformal boundary metric $g_{\mu \nu}^{(0)}$. The local terms denote tensors constructed locally from $g_{\mu \nu}^{(0)}$ and $A_{\mu}^{(0)}$, which may be dropped when computing correlators at non-coincident points.

\section{The purely magnetic brane: zero charge density}

The case of vanishing charge density, with zero or non-zero temperature, provides a physically interesting system, which lends itself to much simpler treatment than the charged case. For this reason, we shall investigate it first here, in its own right.

\subsection{The purely magnetic brane at $T=0$}

We begin with the case of zero temperature. For vanishing charge density and temperature, Lorentz invariance in the $t, x_{3}$ directions is restored. To exhibit this symmetry, it will be convenient to re-interpret $t, x_{3}$ as light-cone coordinates by substituting $t \rightarrow x^{+}$and $x_{3} \rightarrow x^{-}$. In this space-time coordinate system, Lorentz transformations act by $x^{ \pm} \rightarrow \lambda^{ \pm 1} x^{ \pm}$, and further restrict the Ansatz to $E=P=M=N=0$. In terms of the remaining functions $K, L$, the Einstein equations reduce to,

$$
\begin{aligned}
0 & =\left(L^{2} K\right)^{\prime \prime}-24 K \\
0 & =K^{2} L^{\prime \prime}+K K^{\prime} L^{\prime}+2 K K^{\prime \prime} L-L\left(K^{\prime}\right)^{2} \\
-4 B^{2} & =\left(K^{\prime}\right)^{2} L^{2}+4 K K^{\prime} L L^{\prime}+K^{2}\left(L^{\prime}\right)^{2}-24 K^{2}
\end{aligned}
$$

Here, the prime stands for the derivative with respect to $r$. The last equation of 12 is a constraint, whose derivative with respect to $r$ is linear in the first two equations, 
and vanishes, as soon as these equations are satisfied. The first equation gives $L$ as a function of $K$ by quadrature only. Substituting this form of $L$ into the constraint then gives an equation for $K$ which may be solved numerically.

The reduced field equations (12) admit an exact solution for which $K$ is independent of $r$, which is given by,

$$
K(r)=\frac{B}{\sqrt{3}} \quad L(r)=2 \sqrt{3} r
$$

Substitution into the metric of (7) reveals that the corresponding space-time has the form $A d S_{3} \times \mathbf{R}^{2}$, with an $A d S_{3}$ radius given by $\ell_{3}=1 / \sqrt{3}$. The $A d S_{5}$ vacuum, with $K, L \sim r$ is not a solution to the reduced equations (12) when $B \neq 0$, but it does become an approximate solution in the limit of large $r$, namely when $B / r \rightarrow 0$. The $T=0$ purely magnetic brane is the solution to (12) for which the functions $K$ and $L$ tend to the $A d S_{3} \times \mathbf{R}^{2}$ solution of (13) when $r \rightarrow 0$, and is asymptotic to $A d S_{5}$ of (1) when $r \rightarrow \infty$,

$$
K(r) \sim c_{V} r \quad L(r) \sim 2 r
$$

Numerical analysis confirms that such a purely magnetic brane solution exists and is regular for all $r>0$, and gives the numerical value $c_{V}=2.797$.

Fig. 3 The purely magnetic brane solution interpolates between an $A d S_{5}$ space-time with magnetic field for large $r$, and a $A d S_{3} \times R^{2}$ spacetime with magnetic field for small $r$. The holographic dual field theory has zero charge density and temperature.

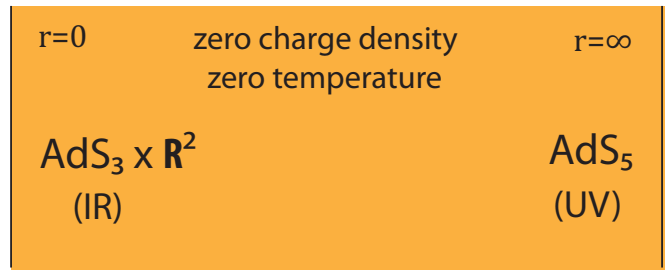

\subsection{RG flow and thermodynamics}

The holographic dual to the purely magnetic brane solution is a renormalization group flow from 3+1-dimensional $\mathscr{N}=4$ super-Yang-Mills theory in the UV (for large $r$ ) to a 1+1-dim. CFT in the IR (for small $r$ ). This flow is schematically represented in figure 3 . The holographic picture is consistent with the qualitative gauge dynamics behavior of strongly interacting Weyl fermions discussed in section 2 The central charge $c$ of this CFT may be derived using the Brown-Henneaux formula [12],

$$
c=\frac{3 \ell_{3}}{2 G_{3}} \quad \frac{1}{G_{3}}=\frac{B V_{2}}{G_{5}}
$$


applied to an $A d S_{3}$ of radius $\ell_{3}=1 / \sqrt{3}$, and where we have taken $x_{1,2}$ to be compactified on a $\mathbf{T}^{2}$ with area $V_{2}$.

The specific heat coefficient at low temperature may be expressed in terms of the entropy density $s$ by $s / T$. In turn, the Cardy formula gives the entropy density $s$ in terms of the central charge of a 1+1-dimensional CFT. It may be used here to extract the holographic specific heat coefficient $s_{\text {grav }} / T$, and the entropy density $s_{\text {grav }}$ in terms of the zero temperature purely magnetic brane, and we find,

$$
\frac{s_{\text {grav }}}{T}=\frac{\pi}{3} c=\frac{\pi B V_{2}}{2 \sqrt{3} G_{5}}=\sqrt{\frac{4}{3}} \frac{s_{\text {gauge }}}{T}
$$

In the last equality, we have included the comparison with the entropy density $s_{\text {gauge }}$ evaluated earlier for free fermions in the lowest Landau level. To exhibit this relation, we have used the AdS/CFT relation $G_{5}=\pi /\left(2 N^{2}\right)$. The fact that the gravity and gauge theory central charges do not agree can be understood as follows [5]. Comparing the central charges at small (but finite) and large values of the 't Hooft coupling should show agreement, because the central charge of a $\mathrm{D}=1+1$ CFT is unchanged under marginal deformation. However, the passage from zero to small 't Hooft coupling can be a discontinuous change if the CFT has a relevant operator that is either absent or present in the two cases. The $\sqrt{4 / 3}$ factor is presumably a result of the appearance of this relevant operator.

To derive the thermodynamics of the purely magnetic brane at all $T$, we replace the near-horizon $A d S_{3} \times \mathbf{R}^{2}$ space by a BTZ $\times \mathbf{R}^{2}$ black brane. The latter is expected to solve the field equations (5) as well since BTZ may be obtained as a quotient of $A d S_{3}$ by a discrete group. Concretely, the absence of electric charge allow us to set $E=P=0$ in 17, but the fields $M, N$ need to be retained at finite temperature. The gauge field is still $F=B d x^{1} \wedge d x^{2}$, while the metric takes the following form,

$$
\begin{array}{ll}
K(r)=\frac{B}{\sqrt{3}} & L(r)=4 \sqrt{3}\left(r-r_{+}\right) \\
N(r)=1 & M(r)=-12\left(r-r_{+}\right)\left(r_{+}-r_{-}\right)
\end{array}
$$

The Hawking temperature is found to be $T=3\left(r_{+}-r_{-}\right) / \pi$. Numerical analysis confirms the existence of a regular solution that interpolates between the above near-horizon BTZ $\times \mathbf{R}^{2}$ solution for small $r$, and asymptotically $A d S_{5}$ for large $r$. Using this pure magnetic brane solution for arbitrary $T$, the entropy density may be

calculated at all $T$, and is found to behave as $s_{\text {grav }} \sim T^{3}$ for high $T$, with the standard factor of $3 / 4$ compared to the high $T$ gauge theory calculation.

\subsection{Calculation of current-current correlators at $T=0$}

The boundary current formalism discussed in section 3.2 may be used to evaluate the various two-point functions of the gauge current $\mathscr{J}^{\mu}$ and the stress tensor $\mathscr{T}^{\mu v}$. 
In the absence of charge density for the purely magnetic brane, the cross correlators $\left\langle\mathscr{J}^{\mu}(x) \mathscr{T}^{\mu \nu}(y)\right\rangle$ will vanish identically. We begin by evaluating the current-current correlators, by combining linear response theory with the formulas of [11,

$$
J^{\mu}(x)=i \int d^{4} y \sqrt{-\operatorname{det}\left(g_{\mu \nu}^{(0)}\right)}\left\langle\mathscr{J}^{\mu}(x) \mathscr{J}^{v}(y)\right\rangle \delta A_{\nu}^{(0)}(y)
$$

Here, the expectation value $J^{\mu}$ of the current $\mathscr{J}^{\mu}$ is sourced by a linear variation in the source $A_{v}^{(0)}$ of the gauge potential, using (11). As no variation of the metric is imposed, the current $J^{\mu}$ may be obtained by linearizing the Maxwell-Chern-Simons equations on the first line of (5) around the purely magnetic brane. We shall be interested in correlators in the 1+1-dimensional effective low energy CFT only, and thus restrict to excitations carrying momentum along the magnetic field direction. The gauge potential for definite momentum is then given by,

$$
A=A_{B}+\left(a_{+}(r, p) d x^{+}+a_{-}(r, p) d x^{-}\right) e^{i p x}
$$

where $d A_{B}=B d x_{1} \wedge d x_{2}$, and we shall use the notations $p x=p_{+} x^{+}+p_{-} x^{-}$and $p^{2}=p_{+} p_{-}$throughout. Denoting the metric fields of the purely magnetic brane solution at $T=0$ by $K$ and $L$, we find that the Maxwell-Chern-Simons equations may be decoupled in terms of the variables $\varepsilon_{ \pm}=p_{-} a_{+} \pm p_{+} a_{-}$for which we obtain the following equations,

$$
\begin{aligned}
& 0=K L\left(K L \varepsilon_{-}^{\prime}\right)^{\prime}-4 k^{2} B^{2} \varepsilon_{-}-2 K^{2} \frac{p^{2}}{L} \varepsilon_{-} \\
& 0=K L \varepsilon_{+}^{\prime}-2 k B \varepsilon_{-}
\end{aligned}
$$

Given that the functions $K$ and $L$ are known only numerically, solving the above equations for general $p^{2}$ can only be achieved numerically. If we restrict attention to the regime of small $p^{2}$, however, then the linearized equations can be solved essentially analytically using the method of overlapping expansions.

\subsection{Method of overlapping expansions}

The characteristic scale of the purely magnetic brane solution, namely where the functional dependence of $K$ and $L$ transits from the behavior of (14) at large $r$ to the behavior of (13) at small $r$, is set by $r \sim 1$.

In the near-region, defined by $r \ll 1$, the purely magnetic brane solution may be approximated by the behavior in (13), so that (20) becomes,

$$
\begin{aligned}
& 0=r^{2} \varepsilon_{-}^{\prime \prime}+r \varepsilon_{-}^{\prime}-k^{2} \varepsilon_{-}-\frac{p^{2}}{12 \sqrt{3} r} \varepsilon_{-} \\
& 0=r \varepsilon_{+}^{\prime}-k \varepsilon_{-}
\end{aligned}
$$


The first equation is of the modified Bessel type and is solved by the Bessel functions $I_{2 k}(p / \sqrt{r})$ and $K_{2 k}(p / \sqrt{r})$. Only the solution $\varepsilon_{-}(r) \sim K_{2 k}(p / \sqrt{r})$ is regular as $r \rightarrow$ 0 , which leads us to reject the solution $I_{2 k}$.

In the far-region, defined by $p^{2} \ll r$, we may neglect the last term of the first equation in 201, and solve the remaining equation in terms of the function,

$$
\psi(r) \equiv \int_{\infty}^{r} \frac{d r^{\prime}}{K\left(r^{\prime}\right) L\left(r^{\prime}\right)}
$$

Note that $\psi(r)$ depends only on the data of the purely magnetic brane solution. Expressing the solution directly in terms of the original variables $a_{ \pm}(r, p)$, we find,

$$
a_{ \pm}=p_{ \pm} \tilde{a}_{0}+\left(a_{ \pm}^{(0)}-p_{ \pm} \tilde{a}_{0}\right) e^{ \pm 2 k B \psi(r)}
$$

where $a_{ \pm}^{(0)}$ and $\tilde{a}_{0}$ are integration constants.

An overlap-region, in which the near-region and the far-region overlap in a finite interval, will exist provided $p^{2} \ll 1$. Assuming that $p^{2} \ll 1$, there will exist an overlap region in which we may match the $p^{2} / r \ll 1$ behavior of the Bessel function in the near-region solution,

$$
a_{ \pm}=C \frac{\left(p^{2} / 12\right)^{\mp k}}{\Gamma(1 \mp 2 k) p_{\mp}} r^{ \pm k}+p_{ \pm} a_{0}
$$

with the $r \ll 1$ behavior of the far-region solution. The latter may be derived from the asymptotic behavior of the function $\psi(r)$, which is found to be for $r \ll 1$,

$$
\psi(r) \sim \frac{1}{2 B} \ln r+\psi_{0}
$$

Numerical evaluation gives $\psi_{0} \approx 0.2625$. Comparing the $r$-dependence in $(24)$ and (23) using 25], we see that the near-region and far-region functional behaviors are indeed the same, and given by a constant term, as well as by $r^{ \pm k}$ terms. Matching these functional dependences produces the full solution.

\subsection{Current two-point correlators}

To complete the calculation of the current-current correlators in the long-distance approximation $p^{2} \ll 1$ we use the overlapping expansion results obtained earlier. The large $r$ approximation for the function $\psi(r)$ is obtained analytically from the asymptotic behavior of $K$ and $L$ in (14),

$$
\psi(r) \sim-\frac{1}{2 c_{V} r}
$$


and used to derive the asymptotic behavior for the gauge potential for $r \rightarrow \infty$,

$$
a_{ \pm}(r, p)=a_{ \pm}^{(0)}+\frac{1}{4 r} a_{ \pm}^{(2)} \quad a_{ \pm}^{(2)}=\mp \frac{4 k B}{c_{V}}\left(a_{ \pm}^{(0)}-p_{ \pm} \tilde{a}_{0}\right)
$$

To obtain $\tilde{a}_{0}$ and $a_{ \pm}^{(2)}$ in terms of $a_{0}$ and $a_{ \pm}^{(0)}$, we match the near-region solution of (24) with the far-region solution of (23). Including proper normalizations [22], we obtain the current-current correlators in the limit $p^{2} \ll 1$,

$$
\begin{aligned}
\left\langle\mathscr{J}_{+}(p) \mathscr{J}_{+}(-p)\right\rangle & =\frac{k c}{2 \pi} \frac{p_{+}}{p_{-}} \frac{1}{1-\zeta p^{4 k}} \\
\left\langle\mathscr{J}_{-}(p) \mathscr{J}_{-}(-p)\right\rangle & =\frac{k c}{2 \pi} \frac{p_{-}}{p_{+}} \frac{\zeta p^{4 k}}{1-\zeta p^{4 k}} \\
\left\langle\mathscr{J}_{+}(p) \mathscr{J}_{-}(-p)\right\rangle & =-\frac{k c}{2 \pi} \frac{\zeta p^{4 k}}{1-\zeta p^{4 k}}
\end{aligned}
$$

where $c$ is the Brown-Henneaux central charge derived in (15), and $\zeta=\zeta(k)$ is a $k$-dependent function whose precise form will not be needed here. We note that the above correlators saturate the chiral anomaly relation independently of $\zeta$,

$$
p_{+} \mathscr{J}_{-}+p_{-} \mathscr{J}_{+}=\frac{k c}{\pi}\left(p_{+} a_{-}^{(0)}-p_{-} a_{+}^{(0)}\right)
$$

To leading order in small $p^{2}$ the correlators involving $\mathscr{J}_{-}$both vanish, while the correlator involving only $\mathscr{J}_{+}$takes the following form in position space,

$$
\left\langle\mathscr{J}_{+}(x) \mathscr{J}_{+}(0)\right\rangle=-\frac{k c}{2 \pi^{2}} \frac{1}{\left(x^{+}\right)^{2}}
$$

With our conventions, the above sign in the central term corresponds to a unitary Abelian Kac-Moody algebra for $k>0$ and $c>0$, as is the case here.

\subsection{Maxwell-Chern-Simons holography in $\mathrm{AdS}_{3}$}

Attempts to formulate Maxwell-Chern-Simons (MCS) holography directly in $\mathrm{AdS}_{3}$ space-time are fraught with subtleties [1]. This circumstance may be investigated directly with the help of the near-region solutions derived in section 4.4 and in particular the large $r$ asymptotics of this solution given in (24),

$$
a_{ \pm}(r)=\alpha_{ \pm}+\beta_{ \pm} r^{ \pm k}
$$

From the point of view directly of $A d S_{3}$, it is unclear which coefficients should be used as sources, and which ones correspond to expectation values. That is, it is not clear which boundary conditions lead to a consistent theory, and indeed most 
boundary conditions lead to problems with instabilities and/or ghosts [1]. Symptoms of this may be detected in the current-current correlators derived in $[29$, by taking the limit $p^{2} \gg 1$. In this limit, the correlators involving the component $\mathscr{J}_{+}$vanish, while the correlator of $\mathscr{J}_{\text {- becomes in position space, }}$

$$
\left\langle\mathscr{J}_{-}(x) \mathscr{J}_{-}(0)\right\rangle=+\frac{k c}{2 \pi^{2}} \frac{1}{\left(x^{-}\right)^{2}}
$$

Although this correlator by itself saturates the chiral anomaly, its sign corresponds to that of a non-unitary Abelian Kac-Moody algebra. This violation of unitarity is a symptom of the disease which besets certain choices of boundary conditions for Maxwell-Chern-Simons directly in $A d S_{3}$.

However, by the same token we see that when the MCS theory is obtained as the IR limit of the holographic RG flow provided by the purely magnetic brane solution from an asymptotic $A d S_{5}$ completion, then the MCS theory makes perfect sense. The key point is that the IR theory comes with a built in UV cutoff, given by the scale at which the $\mathrm{AdS}_{3}$ factor goes over to $\mathrm{AdS}_{5}$. All the would-be inconsistencies are removed by the presence of the UV cutoff.

\subsection{Effective conformal field theory and double-trace operators}

The leading IR contribution to the two-point function of $\mathscr{J}_{+}$in 28 may be parametrized by an effective free scalar field $\phi$ with canonical Lagrangian $\mathscr{L}_{\phi} \sim$ $\partial_{+} \phi \partial_{-} \phi$. The subdominant $p^{4 k}$ terms in the correlators of (28) may be understood in terms of contributions to the Lagrangian from double-trace operators $\mathscr{L}_{\mathscr{O}} \sim \partial_{+} \mathscr{O} \partial_{-} \mathscr{O}$, where $\mathscr{O}$ is a conformal primary field of dimension $(k, k)$. The expressions for the currents and two-point functions (in momentum space) of these operators are given as follows,

$$
\begin{array}{ll}
\mathscr{J}_{+}=\partial_{+} \phi+\partial_{+} \mathscr{O} & \langle\phi(p) \phi(-p)\rangle=p^{-2} \\
\mathscr{J}_{-}=\partial_{-} \mathscr{O} & \langle\mathscr{O}(p) \mathscr{O}(-p)\rangle=\zeta p^{4 k-2}
\end{array}
$$

These two fields summarize the entire IR behavior of the correlators, within our approximations. The importance of double trace operators in the holographic renormalization group [7, 10] has been stressed recently in [38, 28].

We note that the structure of the $\mathscr{J}_{+}$correlators is quite reminiscent of the structure of the charge density two-point function in the Luttinger liquid model. Clearly there is a leading inverse square contribution both in (30) and in (3), while the the $p^{4 k}$ higher order corrections in (28) are analogous to the $x^{-2 \Delta}$ corrections of (3). 


\subsection{Stress tensor correlators and emergent Virasoro symmetry}

Since the purely magnetic brane produces a flow from $A d S_{5}$ towards a space-time containing an $A d S_{3}$ factor, its holographic dual is expected to be a full-fledged CFT in the IR limit, endowed with left- and right-moving Virasoro algebras. This structure, and the value of the associated Brown-Henneaux central charge $c$ of (15) dictate the structure of the two-point function of two stress tensor components. All correlators involving the component $\mathscr{T}_{+-}$vanish at non-coincident points, as does the mixed correlator $\left\langle\mathscr{T}_{++}(x) \mathscr{T}_{--}(y)\right\rangle$. The remaining correlators are given by,

$$
\left\langle\mathscr{T}_{ \pm \pm}(x) \mathscr{T}_{ \pm \pm}(0)\right\rangle=\frac{c}{8 \pi^{2}\left(x^{ \pm}\right)^{4}}
$$

These two-point correlators may be checked by explicit calculation using the method of overlapping expansions along the same lines as for the current correlators, and agree.

The existence of two Virasoro symmetry algebras in the IR brings to light the holographic realization of the emergence of symmetries. Indeed, the BrownHenneaux coordinate transformations on the near-horizon $A d S_{3}$, which produce these Virasoro asymptotic symmetry algebras, correspond to pure gauge transformations. This is as expected, since gravity in three space-time dimensions is (locally) trivial. But these coordinate transformations on $A d S_{3}$ extend to perturbative deformations of the pure magnetic brane solution and interpolate to the $A d S_{5}$ boundary where they correspond to physical deformations which are not merely gauge transformations. Indeed, the asymptotic symmetry algebra at the asymptotically $A d S_{5}$ boundary of the purely magnetic brane is $S O(4,2)$, a finite-dimensional Lie algebra of which the infinite-dimensional Virasoros are certainly not subalgebras. Therefore, we conclude that the Virasoro symmetries present in the IR are emergent symmetries, not present in the UV theory.

\section{Holographic Dual Solutions for non-Zero Charge Density}

A non-zero charge density gives rise to a wealth of interesting physics. As discussed in the introduction, the physical location of the charge, namely either inside or outside the event horizon and a mixture thereof, will to a large extent govern the phase diagram of the dual field theory. Remarkably, it will be possible to understand most of the low temperature dynamics, for large enough magnetic field, using the analytical methods of overlapping expansions, supplemented by a few numerical constants determined from the purely magnetic brane solution. In this section, we shall proceed analytically, and fill in the regions of the phase diagram not accessible through analytical results with the help of numerical results. 


\subsection{Reduced field equations}

Investigating thermodynamics in the presence of charge density and a magnetic field in the $x^{3}$ direction will involve gravitational solutions which are invariant under translations in $x^{\mu}$, and rotations in the $x_{1}, x_{2}$ plane. Thus, we need the full Ansatz of equation (7). The corresponding reduced field equations are as follows,

$$
\begin{array}{ll}
\text { M1 } & \left((N E+L P) e^{2 V}\right)^{\prime}+2 k b P=0 \\
\text { M2 } & \left((L E+M P) e^{2 V}\right)^{\prime}-2 k b E=0 \\
\text { E1 } & L^{\prime \prime}+2 V^{\prime} L^{\prime}+4\left(V^{\prime \prime}+V^{\prime 2}\right) L-4 P E=0 \\
\text { E2 } & M^{\prime \prime}+2 V^{\prime} M^{\prime}+4\left(V^{\prime \prime}+V^{\prime 2}\right) M+4 E^{2}=0 \\
\text { E3 } & N^{\prime \prime}+2 V^{\prime} N^{\prime}+4\left(V^{\prime \prime}+V^{\prime 2}\right) N+4 P^{2}=0 \\
\text { E4 } & f\left(V^{\prime}\right)^{2}+f^{\prime} V^{\prime}+\frac{1}{4}\left(L^{\prime}\right)^{2}-\frac{1}{4} M^{\prime} N^{\prime}+b^{2} e^{-4 V}+M P^{2}+2 L E P+N E^{2}=6 \\
\mathrm{fV} & \left(f e^{2 V}\right)^{\prime \prime}=24 e^{2 V}
\end{array}
$$

We have used the notation $f=L^{2}-M N$ of (8), and changed variables to $K=e^{2 V}$. Also, we now denote the magnetic field as $b$, reserving the use of $B$ for the value of the magnetic field in a canonical coordinate system.

The reduced field equations admit a number of first integrals. Using the potentials $A$ and $C$ for $E=A^{\prime}$ and $P=-C^{\prime}$, equations M1 and M2 admit obvious first integrals,

$$
\begin{aligned}
\left(N A^{\prime}-L C^{\prime}\right) e^{2 V}+2 k b C & =0 \\
\left(L A^{\prime}-M C^{\prime}\right) e^{2 V}-2 k b A & =0
\end{aligned}
$$

The integration constants to $A$ and $C$ that arise here have been absorbed into the definition of these functions. Forming combinations of equations E1, E2, and E3, and using (36), we find the following further first integrals,

$$
\begin{array}{rlrl}
\lambda e^{2 V}-4 k b A C & =\lambda_{0} & 2 \lambda & =N M^{\prime}-M N^{\prime} \\
\mu e^{2 V}+4 k b A^{2} & =\mu_{0} & \mu & =L M^{\prime}-M L^{\prime} \\
v e^{2 V}+4 k b C^{2} & =v_{0} & v & =N L^{\prime}-L N^{\prime}
\end{array}
$$

where $\lambda_{0}, \mu_{0}$, and $v_{0}$ are the constant values of the corresponding first integrals. Equation $\mathrm{fV}$ is linear in $f$ and may be solved for as a function of $V$. Finally, $\lambda, \mu, v$ satisfy a purely kinematic relation,

$$
\left(f^{\prime}\right)^{2}=4\left(\lambda^{2}-\mu v\right)+4 f\left(L^{\prime}\right)^{2}-4 f M^{\prime} N^{\prime}
$$

Under constant $\Lambda \in S L(2, \mathbf{R})$ transformations of the coordinates $x^{ \pm}$,

$$
\left(\begin{array}{c}
\tilde{x}^{+} \\
\tilde{x}^{-}
\end{array}\right)=\Lambda^{-1}\left(\begin{array}{l}
x^{+} \\
x^{-}
\end{array}\right)
$$


the Ansatz (7), the reduced field equations (35), and the first integrals (36) and (37) are invariant provided the fields transform as,

$$
\left(\begin{array}{c}
\tilde{A} \\
-\tilde{C}
\end{array}\right)=\Lambda^{t}\left(\begin{array}{c}
A \\
-C
\end{array}\right) \quad\left(\begin{array}{cc}
\tilde{M} & \tilde{L} \\
\tilde{L} & \tilde{N}
\end{array}\right)=\Lambda^{t}\left(\begin{array}{cc}
M & L \\
L & N
\end{array}\right) \Lambda
$$

while the field $V$ and the combination $f$ are invariant. The triplet $(\lambda, \mu, v)$ is the $S L(2, \mathbf{R})$ analogue of angular momentum and transforms under the vector representation of $S L(2, \mathbf{R})$, just as their first integral values $\left(\lambda_{0}, \mu_{0}, v_{0}\right)$ do.

\subsection{Near-horizon Schrödinger geometry}

Introducing charge requires that $E \neq 0$ in the Ansatz of (7). Re-interpreting $t$ as $x^{+}$ and $x_{3}$ as $x^{-}$, we see that turning on a charge corresponds to a deformation which is null in the $x^{ \pm}$coordinate system. This suggests the existence of a solution in which deformations in the $x^{-}$directions vanish. We begin by exhibiting an exact charged near-horizon solution in which the $x_{1}, x_{2}$-directions are frozen out by the presence of a magnetic field, so that $K=e^{2 V}$ is constant. The gauge potential and electric field are found as follows,

$$
A(r)=\frac{e_{0}}{k} r^{k} \quad E(r)=e_{0} r^{k-1}
$$

The near-horizon metric takes the form,

$$
d s^{2}=\frac{d r^{2}}{12 r^{2}}+4 \sqrt{3} r d t d x_{3}-\left(\alpha_{0} r+\frac{2 e_{0}^{2} r^{2 k}}{k(2 k-1)}\right) d t^{2}+d x_{1}^{2}+d x_{2}^{2}
$$

In these coordinates we have $b=\sqrt{3}$. This metric coincides with the Schrödinger space-time of [8, 60] and the null-warped solution of [6].

\subsection{The charged magnetic brane solution}

The near-horizon Schrödinger geometry at $r \rightarrow 0$ extends to a regular charged magnetic brane solution to the full reduced field equations (35) with asymptotic $A d S_{5}$ behavior. In this respect, the role played by the Schrödinger near-horizon geometry for the charged magnetic brane is parallel to the role played by the $A d S_{3} \times \mathbf{R}^{2}$ near-horizon geometry of the purely magnetic brane. The full solution has vanishing deformations in the $x^{-}$direction, so that we can set $C=N=0$, and the functions $K=e^{2 V}$ and $L$ remain those of the purely magnetic brane. The remaining fields $A$ and $M$ may be obtained by quadrature from $K=e^{2 V}$ and $L$, and we find,

$$
A(r)=A_{\infty} e^{2 k b \psi(r)}
$$




$$
M(r)=L(r)\left(-\frac{\alpha_{\infty}}{2 \sqrt{3}}-4 k b \int_{\infty}^{r} \frac{d r^{\prime} A\left(r^{\prime}\right)^{2}}{K\left(r^{\prime}\right) L\left(r^{\prime}\right)^{2}}\right)
$$

The function $\psi(r)$ was defined in (22). Using the $r \rightarrow 0$ asymptotics of $\psi(r)$ given in (25), we see that the gauge potential satisfies the standard regularity condition $A(0)=0$ at the horizon. Using the $r \rightarrow \infty$ asymptotics of $[26$, we see that the integration constant $A_{\infty}=A(\infty)$ is the chemical potential. The integration constant $\alpha_{\infty}$ introduces a relative tilt between the light-cones in the UV and the IR. Solutions for different values of $A_{\infty}$ and $\alpha_{\infty}$ are related to one another by $\operatorname{SL}(2, \mathbf{R})$ transformations which preserve the restrictions $C=N=0$, and we have,

$$
\Lambda=\left(\begin{array}{cc}
\lambda_{1} & 0 \\
\lambda_{2} & \lambda_{1}^{-1}
\end{array}\right) \quad \begin{array}{ccc}
\tilde{A}_{\infty} & = & \lambda_{1} A_{\infty} \\
\tilde{\alpha}_{\infty} & =\lambda_{1}^{2} \alpha_{\infty}-2 \lambda_{1} \lambda_{2}
\end{array}
$$

Therefore, all solutions with $A_{\infty} \neq 0$ are equivalent to one another under $S L(2, \mathbf{R})$.

The asymptotic behavior near the boundary of $A d S_{5}$ is given as follows,

$$
\begin{array}{ll}
A(r) \sim A_{\infty}-\frac{c_{E}}{r} & c_{E}=\frac{k b A_{\infty}}{c_{V}} \\
M(r) & \sim-\frac{\alpha_{\infty}}{\sqrt{3}} r
\end{array}
$$

The integration constants $e_{0}$ and $\alpha_{0}$ of the near-horizon Schrödinger geometry may be related to the parameters $\alpha_{\infty}$ and $A_{\infty}$ of the boundary, and we find,

$$
\begin{aligned}
& A_{\infty}=e_{0} e^{-2 k b \psi_{0}} \\
& \alpha_{\infty}=\alpha_{0}+16 c_{V}^{2} c_{E}^{2} J(k) \quad J(k)=\frac{1}{2 k} \int_{0}^{\infty} d r \frac{e^{4 k b \psi(r)}}{K(r) L(r)^{2}}
\end{aligned}
$$

where the constant $\psi_{0}$ was defined in 25 .

\subsection{Regularity of the solutions}

In anticipation of extending the charged magnetic brane solution to finite temperature, we must require regularity of the solution as a black brane. Thus, the coefficient function of $d t^{2}$ in the metric must remain negative throughout the space-time region outside the (outer) horizon, which leads us to require,

$$
M(r) \leq 0
$$

with equality only at the horizon $r=0$. At infinity, this imposes the condition $\alpha_{\infty}>$ 0 . The solution near the horizon of (42) imposes further conditions which depend on the value of $k$. In the parameter region $0 \leq k<1 / 2$, the $r^{2 k}$ term dominates over the $\alpha_{0} r$ term, and leads to $M(r)>0$ as soon as $e_{0} \neq 0$. Thus, the charged magnetic 
brane solution in the region $0 \leq k<1 / 2$ is excluded, as it cannot arise as the zero temperature limit of a nonsingular finite temperature black brane.

In the parameter region $1 / 2<k$, it is the $\alpha_{0} r$ term that dominates, which requires $\alpha_{0} \geq 0$. The value $\alpha_{0}=0$ is actually regular as well, since the $r^{2 k}$ term contributes negatively for $1 / 2<k$. It is straightforward to see from (43) that these conditions are also sufficient to make the charged magnetic brane solution regular for all $0<r<\infty$.

\subsection{Existence of a critical magnetic field}

The regularity conditions derived in the preceding section on the parameters $\alpha_{0}$ and $\alpha_{\infty}$ may be translated into conditions on physically observable parameters in the dual field theory. Since the boundary field theory is conformal invariant, only dimensionless combinations of data can enjoy physical meaning. The magnetic field $B$ and the charge density $\rho$ have non-trivial dimension, but the ratio defined by,

$$
\hat{B} \equiv \frac{B}{\rho^{2 / 3}}
$$

is dimensionless, and physically observable. Expressions for $B$ and $\rho$, which denote the values of the magnetic field and charge density in coordinates such that the $\mathrm{AdS}_{5}$ metric takes a canonical for, may themselves may be read off from the boundary behavior of the solution, and are given by,

$$
B=\frac{2 b}{c_{V}} \quad \rho=4 c_{E} \sqrt{\frac{2 b}{\alpha_{\infty}}}
$$

where $c_{E}$ was defined in 45 . Thus, $\hat{B}^{3}$ may be cast in the following form,

$$
\hat{B}^{3}=\frac{3 \alpha_{\infty}}{4 c_{V}^{3} c_{E}^{2}} \quad \hat{B}_{c}^{3} \equiv \frac{3\left(\alpha_{\infty}-\alpha_{0}\right)}{4 c_{V}^{3} c_{E}^{2}}=\frac{12 J(k)}{c_{V}}
$$

Here, we have also defined the combination $\hat{B}_{c}$ in terms of which we obtain the following final expression for $\hat{B}$,

$$
\frac{\hat{B}_{c}^{3}}{\hat{B}^{3}}=1-\frac{\alpha_{0}}{\alpha_{\infty}}
$$

Positivity of $J(k)$ for $k>0$ implies $\alpha_{0}<\alpha_{\infty}$, while regularity required $0 \leq \alpha_{0}$. Thus, we conclude that the charged magnetic brane solution obtained above is regular if and only if $\hat{B}_{c} \leq \hat{B}$. In this sense, $\hat{B}_{c}$ represents a critical magnetic field. Its value depends only on the CS coupling $k$ and the data of the purely magnetic brane solution. Inspection of the behavior of $L$ and $\psi$ in the integral for $J(k)$ shows that $J(k)$, and hence $\hat{B}_{c}$ diverges as $k \rightarrow 1 / 2$, thus providing a natural physical end point for the validity of the charged magnetic brane solution. 


\subsection{Low T thermodynamics for $\hat{B}>\hat{B}_{c}$}

The low $T$ behavior dual to the charged magnetic black brane solution must be investigated separately for magnetic fields $\hat{B}>\hat{B}_{c}$ and $\hat{B} \sim \hat{B}_{c}$. We begin here with the study of the former. The presence of a low non-zero temperature induces only small changes to the charged magnetic brane solution for large $r$, while substantially altering its near-horizon behavior. The corresponding leading $T$-dependent nearhorizon behavior needs to be treated exactly to incorporate these effects.

Our starting point is the purely magnetic BTZ $\times \mathbf{R}^{2}$ solution already discussed in section 4.2. Its metric is given by (17), but it will be convenient here to choose the outer horizon at $r=0$, so that $r_{+}=0$, and to parametrize the solution as follows,

$$
\begin{aligned}
F & =b d x^{1} \wedge d x^{2} \\
d s^{2} & =\frac{d r^{2}}{12 r^{2}+m n r}-m r d t^{2}+4 \sqrt{3} d t d x_{3}+n d x_{3}^{2}+d x_{1}^{2}+d x_{2}^{2}
\end{aligned}
$$

For an asymptotically $A d S_{5}$ space-time given by,

$$
d s^{2}=\frac{d r^{2}}{4 r^{2}}-\frac{\alpha_{\infty}}{\sqrt{3}} r d t^{2}+4 r d t d x_{3}+c_{V}\left(d x_{1}^{2}+d x_{2}^{2}\right)
$$

the dimensionless form of the entropy density $\hat{s}$, and of the temperature $\hat{T}$ may be expressed as follows,

$$
\hat{s} \equiv \frac{s}{B^{3 / 2}}=\frac{\sqrt{n c_{V} \alpha_{\infty}}}{24} \quad \hat{T} \equiv \frac{T}{B^{1 / 2}}=\frac{m \sqrt{n c_{V}}}{4 \pi \sqrt{\alpha_{\infty}}}
$$

In their ratio all reference to $n$ cancels out,

$$
\frac{\hat{s}}{\hat{T}}=\frac{\pi}{6} \frac{\alpha_{\infty}}{m}
$$

This ratio has a finite limit as $T \rightarrow 0$, and may be evaluated in terms of the data of the $T=0$ charged magnetic solution, for which we have $m=\alpha_{0}$ and $n=0$ by (42). Along with the result for $\alpha_{0} / \alpha_{\infty}$ from (51), we find a remarkably simple formula,

$$
\frac{\hat{s}}{\hat{T}}=\frac{\pi}{6} \frac{\hat{B}^{3}}{\hat{B}^{3}-\hat{B}_{c}^{3}}
$$

A number of remarks are in order.

1. In our system, the physical entropy density vanishes at zero temperature (in contrast with the non-vanishing entropy density used in [48, 27, 13] in $2+1$ dimensions.

2. The limit $\hat{B} \rightarrow \infty$ corresponds to vanishing charge density $\rho$ at fixed $B$, and reproduces the zero charge density result of 16 . 
3. The dependence on $\hat{B} / \hat{B}_{c}$ manifested in (56) is reminiscent of the dependence on the excitation velocity and couplings in the Luttinger liquid theory in (3).

4. The divergence of $\hat{s} / \hat{T}$ at zero temperature as $\hat{B} \rightarrow \hat{B}_{c}$ signals the presence of a quantum critical point at $\hat{B}_{c}$. Therefore, in a small region around $T=0$ and $\hat{B}=\hat{B}_{c}$ in the $\hat{T}, \hat{B}$ plane, we should expect to find quantum critical behavior, to be explored in the subsequent subsections.

5. The phase for $\hat{B}<\hat{B}_{c}$ has non-zero entropy density at $\hat{T}=0$, and may be thought of as a deformation of the Reissner-Nordstrom solution for zero magnetic field.

6. Numerical solutions perfectly reproduce the above analytical approximations, as will be explained in section 5.9 .

\subsection{Low Thermodynamics for $\hat{B}=\hat{B}_{c}$}

Precisely at the quantum critical point, we have $\hat{B}=\hat{B}_{c}$, or equivalently $\alpha_{0}=0$. The resulting near-horizon metric of (42) then simplifies slightly,

$$
d s^{2}=\frac{d r^{2}}{12 r^{2}}+4 \sqrt{3} r d t d x_{3}-\frac{2 e_{0}^{2} r^{2 k}}{k(2 k-1)} d t^{2}+d x_{1}^{2}+d x_{2}^{2}
$$

More importantly, however, the metric now is invariant under the following scaling transformations,

$$
r \rightarrow \lambda r \quad t \rightarrow \lambda^{-k} t \quad x_{3} \rightarrow \lambda^{k-1} x_{3}
$$

with $x_{1}, x_{2}$ unchanged. The associated dynamical scaling exponent is given by,

$$
z=\frac{k}{1-k}
$$

General arguments show that, for fixed $\hat{B}=\hat{B}_{c}$, the entropy density scales with temperature according to the relation $\hat{s} \sim \hat{T}^{d / z}=T^{1 / z}$ given that the scaling theory here has space dimension $d=1$.

Numerical analysis shows that the above prediction, based on the structure of the near-horizon metric and its scaling symmetry, is borne out in only a limited range for $k$. The actual behavior is as follows,

$$
\begin{array}{ll}
\hat{s} \sim \hat{T}^{(1-k) / k} & 1 / 2<k<3 / 4 \\
\hat{s} \sim \hat{T}^{1 / 3} & 3 / 4<k
\end{array}
$$

The numerical accuracy for the exponents is better than $1 \%$ for the points we have checked. Note that the exponent matches continuously across the value $k=3 / 4$. Inspection of the numerically obtained metric functions shows that the near-horizon region for finite $\hat{T}$ holds an electrically charged black brane whose space-time metric differs from that of BTZ. 
To understand why the arguments based on the scaling symmetry of the nearhorizon metric fail for $k>3 / 4$, we consider a scaling transformation which leaves the $A d S_{3}$ part of the metric invariant, but not necessarily the term in $e_{0}^{2}$. For example, applying the following scaling,

$$
r \rightarrow \lambda r \quad t \rightarrow t / \sqrt{\lambda} \quad x_{3} \rightarrow x_{3} / \sqrt{\lambda}
$$

with $x_{1}, x_{2}$ unchanged, will scale the term in $e_{0}^{2}$ by a factor of $\lambda^{2 k-1}$. Scaling towards the IR corresponds to $\lambda<1$, and we see that the term in $e_{0}^{2}$ naively becomes irrelevant. Whether the term indeed is irrelevant becomes a dynamical question, which is not easy to settle. Detailed arguments were given in [19] that the separation point is indeed $k=3 / 4$. The scaling exponent of $1 / 3$ may be reproduced for the range $k>1$ with the help of the method of overlapping expansions. The calculations are technically involved, and will not be reproduced here.

In figure 4 we display numerical data illustrating the crossover from the behavior $\hat{s} \sim \hat{T}$ to the behavior $\hat{s} \sim \hat{T}^{1 / 3}$ for $k=k_{\text {susy }}=2 / \sqrt{3}$. Qualitatively, the cross-over behavior of figure 4 persists for all $k>3 / 4$.

\subsection{Scaling function in the quantum critical region}

In a small region surrounding the quantum critical point $T=0$ and $\hat{B}=\hat{B}_{c}$, a critical scaling regime sets in. For the range $k>1$, we have been able to derive this scaling behavior with the help of the method of overlapping expansions, and we find,

$$
\hat{s}=\hat{T}^{1 / 3} f\left(\frac{\hat{B}-\hat{B}_{c}}{\hat{T}^{2 / 3}}\right)
$$

for a certain scaling function $f$ (which is not to be confused with the metric function $f$ introduced in (35)). At $\hat{B}=\hat{B}_{c}$, this formula reproduces the scaling behavior discussed in (60) of the previous section for $k>1$. For $\hat{B}>\hat{B}_{c}$, and low temperature, namely $\hat{T}^{2 / 3} \ll\left(\hat{B}-\hat{B}_{c}\right)$, we should recover (56), so that we should have $f(x) \sim \pi \hat{B}_{c} /(18 x)$ for large $x$. Actually, the method of overlapping expansions allows one to compute $f(x)$ for the range $k>1$, and we shall quote here the result without reproducing the derivation given in [19],

$$
f(x)\left(f(x)^{2}+\frac{x}{32 k \hat{B}_{c}^{4}}\right)=\frac{\pi}{576 k \hat{B}_{c}^{3}}
$$

The scaling function $f$ continues to apply for $\hat{B}<\hat{B}_{c}$, and we find the following behavior for the entropy density as a function of the magnetic field,

$$
\hat{s}=\frac{\sqrt{\hat{B}_{c}-\hat{B}}}{4 \sqrt{2 k} \hat{B}_{c}^{2}}
$$


The value of the exponent is reproduced to approximately $0.2 \%$ accuracy by numerical simulations, and the prefactor to approximately $20 \%$.

\subsection{Numerical completion of the holographic phase diagram}

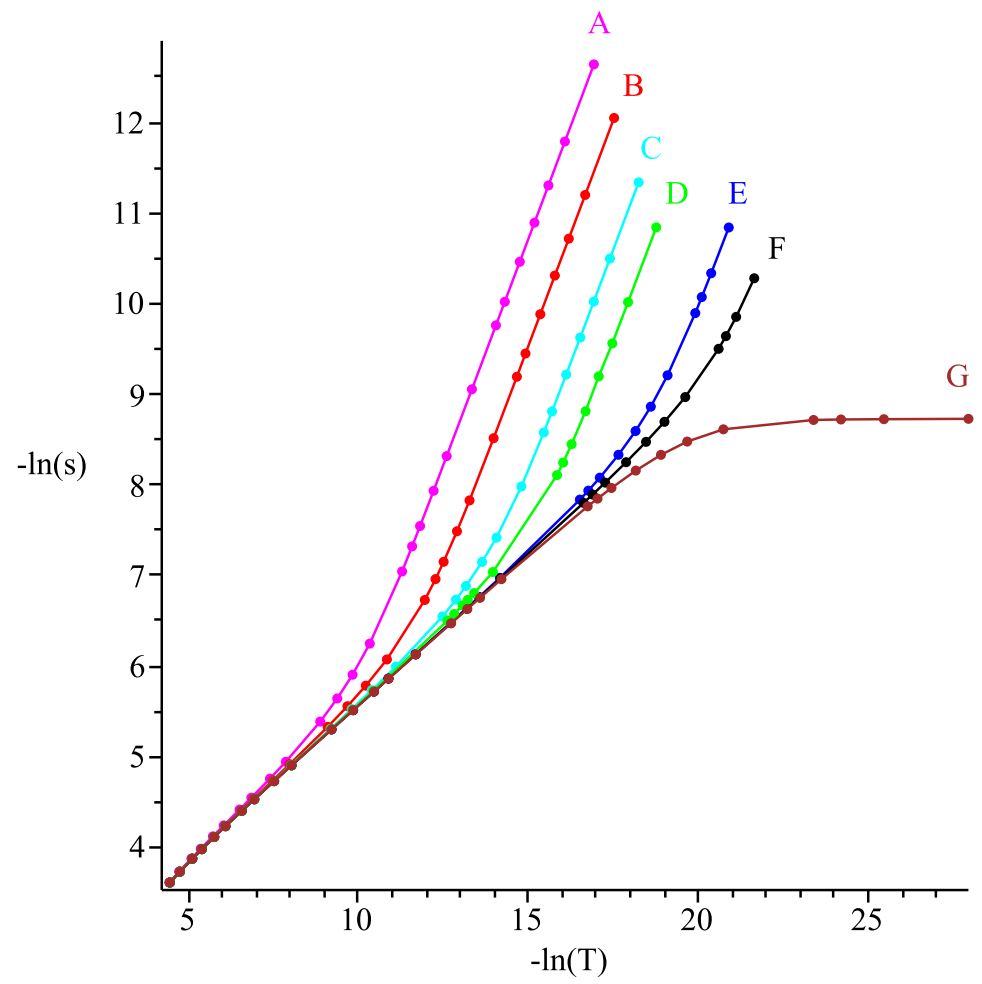

Fig. 4 The cross-over behavior of $\hat{s}$ versus $\hat{T}$ for $k=k_{\text {susy }}=2 / \sqrt{3}$ at values of $\hat{B}>\hat{B}_{c}$ corresponding to the curves $A: \hat{B}^{3}=0.125, B: \hat{B}^{3}=0.1247, C: \hat{B}^{3}=0.1246, D: \hat{B}^{3}=0.12458$, $E: \hat{B}^{3}=0.12457, F: \hat{B}^{3}=0.124569$, and $G: \hat{B}^{3}=0.124568$. To lighten notations, hats on the variables $\hat{T}, \hat{s}$ have not been exhibited in labeling the figure. At moderately low temperatures, $\hat{s}$ scales as $\hat{T}^{1 / 3}$ (lower left corner), while at ultra-low temperatures $\hat{s}$ scales as $\hat{T}$ for $\hat{B}>\hat{B}_{c}$ (curves $A, B, C, D, E, F$ ) and tends to a non-zero constant for $\hat{B}<\hat{B}_{c}$ (curve $G$ ). The dots represent numerical data points, while the solid interpolating lines are included to guide the eye.

The full holographic phase diagram in the variables $\hat{B}, \hat{T}$ is presented in figure 5 Here, the various asymptotic behaviors are combined onto a single graph for the range $k>3 / 4$. For the range $1 / 2<k<3 / 4$, the scaling exponent $1 / 3$ at $\hat{B}=\hat{B}_{c}$ 
must be replaced by $(1-k) / k$, and the behavior of the entropy density for $\hat{B}<\hat{B}_{c}$ is altered though we have not systematically studied the corresponding modifications.

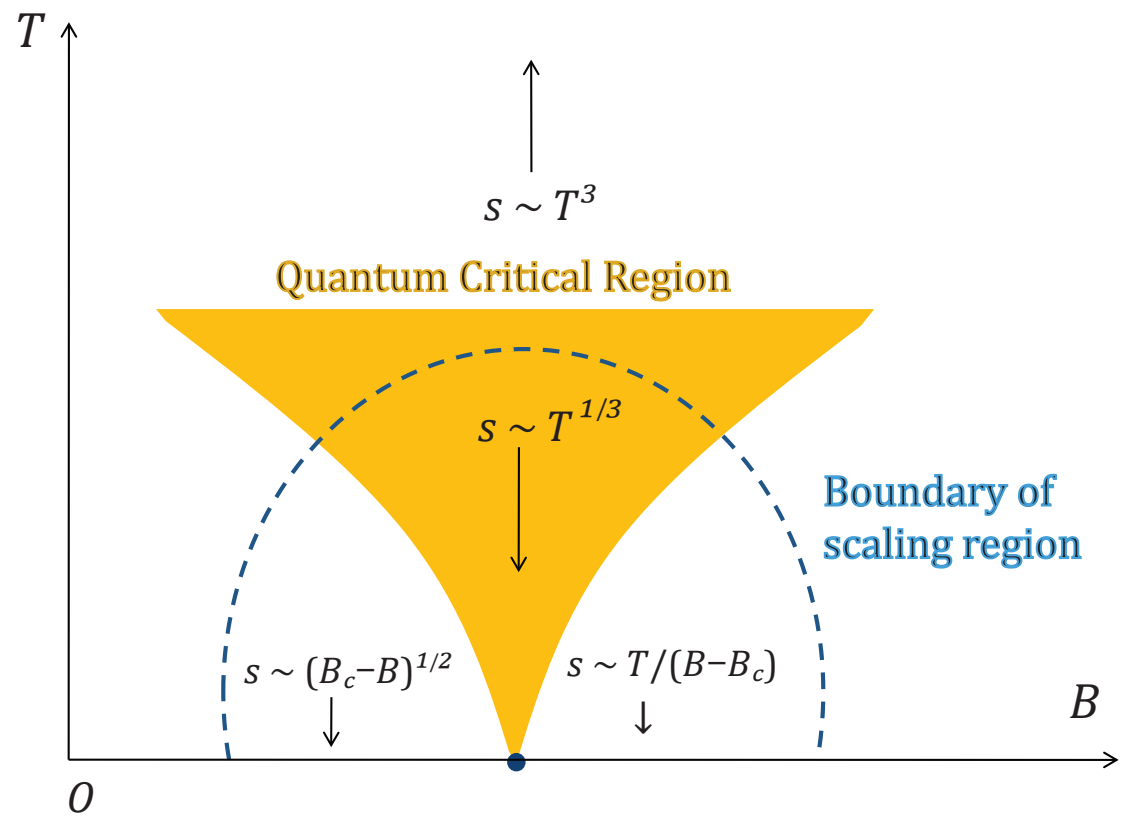

Fig. 5 The full holographic phase diagram in terms of the variables $\hat{B}$ and $\hat{T}$ for $k>3 / 4$. Hats on $\hat{B}, \hat{T}, \hat{s}$, and $\hat{B}_{c}$ have not been exhibited in the figure.

\subsection{Correlators at non-zero charge density}

Correlators of the Maxwell current $\mathscr{J}^{\mu}$ and of the stress tensor $\mathscr{T}^{\mu v}$ may be evaluated, in the long distance approximation, in the presence of a magnetic field at zero temperature, but now with non-vanishing background electric charge density $\rho$, or equivalently, with chemical potential $\mu$. As in the case with vanishing charge density studied in sections 4.3, 4.5, and 4.8, we use the method of overlapping expansions of 4.4 valid for $k>1$. The calculations for the charged case proceed in analogy with the ones for the neutral case, but are now considerably more delicate and technically involved. We refer to the original paper [19] for their detailed derivation, and restrict here to quoting and explaining the results.

Correlators involving the operators with minus chirality are unmodified from the zero charge case. In particular, the two point function of $\mathscr{J}_{-}$has no singularities, while the two point function of $\mathscr{T}_{--}$continues to be given by (34). The correlators with plus chirality are found as follows, 


$$
\begin{aligned}
\left\langle\mathscr{J}_{+}(x) \mathscr{J}_{+}(0)\right\rangle & =-\frac{k c}{2 \pi^{2}} \frac{1}{\left(x^{+}\right)^{2}} \\
\left\langle\mathscr{J}_{+}(x) \mathscr{T}_{++}(0)\right\rangle & =+\frac{k c \mu}{2 \pi^{2}} \frac{1}{\left(x^{+}\right)^{2}} \\
\left\langle\mathscr{T}_{++}(x) \mathscr{T}_{++}(0)\right\rangle & =-\frac{k c \mu^{2}}{2 \pi^{2}} \frac{1}{\left(x^{+}\right)^{2}}+\frac{c}{8 \pi^{2}\left(x^{+}\right)^{4}}
\end{aligned}
$$

where $\mu$ is the chemical potential, related to the charge density by,

$$
\mu=A_{\infty}=\frac{\rho c_{V} \sqrt{\alpha_{\infty}}}{4 k b \sqrt{2 b}}
$$

The system of correlators in the presence of charge may be related to the system of correlators of operators $\mathscr{J}_{+}^{(0)}$ and $\mathscr{T}_{++}^{(0)}$ at zero charge density by the following simple operator mixing relations,

$$
\begin{aligned}
\mathscr{J}_{+} & =\mathscr{J}_{+}^{(0)} \\
\mathscr{T}_{++} & =\mathscr{T}_{++}^{(0)}-\mu_{+} \mathscr{J}_{+}^{(0)}
\end{aligned}
$$

Here, we have exhibited the natural Lorentz weight of the chemical potential by setting $\mu=\mu_{+}$. We see that the underlying Abelian Kac-Moody algebra for $\mathscr{J}_{+}^{(0)}$ and the underlying Virasoro algebras for $\mathscr{T}_{ \pm \pm}^{(0)}$ are unmodified, with unchanged KacMoody level $k c$, and Virasoro central charge $c$.

\subsection{Comments on stability}

The solutions studied here can, at least for special values of $k$, be uplifted to full solutions of higher dimensional supergravity and string theory, but nothing guarantees that they are stable solutions. There are two types of potential instabilities to be aware of: those coming from fields already included in our analysis, and those required by a consistent embedding into supergravity/string theory. Regarding the former, it has been observed in several contexts that the combination of electric charge and Chern-Simons terms can lead to instabilities towards spatially modulated phases [23, 53]. In some cases new solutions with reduced symmetry can be found [24, 41, 25, 25, 42]. As for the latter, a supergravity/string theory embedding will typically bring along a variety of charged fields, and these may be unstable towards forming a condensate, as in holographic superconductors [36]. It is clearly an important challenge to determine when our solutions are unstable, and if not, to characterize the nature of the true ground state. 


\section{Quantum Criticality in 2+1 dimensions}

The critical theories studied so far originate from an underlying 3+1-dimensional gauge theory in the UV which flows towards an effective 1+1-dimensional strongly interacting CFT in the IR. Low temperature thermodynamics and long-distance correlators all signal massless propagation along the direction parallel to the magnetic field only. In the gravity dual, this IR behavior results from the existence of a near-horizon Schrödinger geometry of the form $\mathrm{WAdS}_{3} \times \mathbf{R}^{2}$, where $\mathrm{WAdS}_{3}$ is a null-warped deformation of $A d S_{3}$ space-time. The physical mechanism driving the quantum critical transition on the gravity side is the gradual expulsion of electric charge from the inside of the black brane horizon to the outside of the horizon as the magnetic field $\hat{B}=B / \rho^{2 / 3}$ is being increased; see [37] for another example of this phenomenon.

While quantum criticality in $1+1$ dimensions is certainly of considerable physical interest, as was pointed out in the preceding section, it is probably even more urgent to extend the study to higher dimensions. Quantum criticality in $2+1$ dimensions is relevant to the physics of layered materials, such as cuprates, and graphene. In the present section, we shall exhibit quantum critical behavior in $2+1$ dimensions systems in the presence of a magnetic field, and a non-vanishing electric charge density by holographic methods. Criticality here is driven by the same holographic mechanism that governed the 1+1-dimensional case, namely charge expulsion from the black brane horizon. This time, however, the IR behavior in the gravity dual results from a flow from $A d S_{6}$ in the UV to a near-horizon Lifshitz geometry [44] in the IR which is a deformation of $A d S_{4}$. See [33, 11] for other examples of holographic RG flows involving Lifshitz spacetime. The $\mathrm{AdS}_{6}$ geometry in the UV should be thought of as being dual to some $5+1$ dimensional CFT, examples of which do exist, and have been identified in [59].

\subsection{Field equations and structure of the solutions}

The charge expulsion mechanism operating in the flow from $A d S_{5}$ to deformations of $A d S_{3}$ is made possible by the presence of the Chern-Simons interaction for the Maxwell field, and the existence of the transition crucially depends upon the strength of the associated Chern-Simons coupling $k$. This is because the Chern-Simons term provides the mechanism by which the bulk gauge field can carry its own charge.

The charge expulsion mechanism in higher dimensions that we shall focus on will also be made possible by the presence of Chern-Simons terms. Starting with $A d S_{6}$ in the UV does not support a Chern-Simons terms for the bulk gauge field all by itself. Thus, we are led to introducing further form fields. In the simplest extension, we add a single two-form potential $C$ with field strength $G=d C$. The corresponding Einstein-Maxwell-Chern-Simons action then becomes, 


$$
\begin{aligned}
S & =-\frac{1}{16 \pi G_{6}} \int d^{6} x \sqrt{g}\left(R-\frac{20}{\ell^{2}}+F_{M N} F^{M N}+\frac{1}{3} G_{M N P} G^{M N P}\right)+S_{C S} \\
S_{C S} & =\frac{k}{4 \pi G_{6}} \int C \wedge F \wedge F
\end{aligned}
$$

where $G_{6}$ is the 5+1-dimensional Newton constant, $F=d A$ is the Maxwell field strength, and $-20 / \ell^{2}$ stands for the cosmological constant for an asymptotic $A d S_{6}$ vacuum solution of radius $\ell$, which we shall set to 1 . Boundary and counter term contributions to the action are not being exhibited here.

The Maxwell-Chern-Simons field equations are,

$$
\begin{aligned}
d * F-2 k F \wedge G & =0 \\
d * G+k F \wedge F & =0
\end{aligned}
$$

while the Einstein equations are,

$$
R_{M N}=-2 F_{M P} F_{N}^{P}-G_{M P Q} G_{N}{ }^{P Q}+g_{M N}\left(5+\frac{1}{4} F_{P Q} F^{P Q}+\frac{1}{6} G_{P Q R} G^{P Q R}\right)
$$

Clearly, the charge densities for both the $F$ and $G$ fields are proportional to the Chern-Simons coupling $k$.

As we focus here on thermodynamic questions, we shall be interested in solutions which are invariant under translations in $x^{\mu}=\left(t, x_{1}, x_{2}, x_{3}, x_{4}\right)$. The flow from $A d S_{6}$ in the UV to $A d S_{4}$ and its deformations in the IR will be generated by a constant magnetic field, which we shall choose in the direction $F_{34}=B$. It is natural to require rotation invariance in the $x_{3}, x_{4}$ plane, as well as in the remaining space directions $x_{1}, x_{2}$. A general Ansatz invariant under these symmetries was constructed in [21], and is given by,

$$
\begin{aligned}
F & =E d r \wedge d t+\tilde{B} d x_{1} \wedge d x_{2}+B d x_{3} \wedge d x_{4} \\
G & =\left(G_{1} d r+G_{2} d t\right) \wedge d x_{1} \wedge d x_{2}+\left(G_{3} d r+G_{4} d t\right) \wedge d x_{3} \wedge d x_{4} \\
d s^{2} & =\frac{d r^{2}}{U}-U d t^{2}+e^{2 V_{1}}\left(d x_{1}^{2}+d x_{2}^{2}\right)+e^{2 V_{2}}\left(d x_{3}^{2}+d x_{4}^{2}\right)
\end{aligned}
$$

By translation invariance in $x^{\mu}$, the coefficients $B, \tilde{B}, E, G_{1}, G_{2}, G_{3}, G_{4}, U, V_{1}, V_{2}$ depend only on $r$. By the Bianchi identities for $F$ and $G$, the quantities $B, \tilde{B}, G_{2}, G_{4}$ must actually be independent of $r$. The magnetic field $\tilde{B}$ plays the role of a magnetic field living in the IR 2+1-dimensional field theory, and will be set to zero here for simplicity, $\tilde{B}=0$. For $k B \neq 0$, the field equations then imply that $G_{2}=0$ and $G_{3} G_{4}=0$. Solutions with either $G_{3} \neq 0$ or $G_{4} \neq 0$ do not have regular horizons, so we set also $G_{3}=G_{4}=0$. The remaining reduced field equations were derived in [21], and will not be repeated here as they are reasonably involved. 


\subsection{Horizon and asymptotic data, physical quantities}

We choose a coordinate $r$ such that the horizon is at $r=0$. We normalize the scales of the coordinates $x^{\mu}$ by setting,

$$
U(0)=V_{1}(0)=V_{2}(0)=0 \quad U^{\prime}(0)=1
$$

The field equations relate the horizon values $G_{1}(0)=-2 k b E(0)$. The asymptotic behavior for $r \rightarrow \infty$ may be parametrized analogously,

$$
U(r) \sim r^{2} \quad e^{2 V_{1}(r)} \sim v_{1} r^{2} \quad e^{2 V_{2}(r)} \sim v_{2} r^{2}
$$

The asymptotics of the gauge field strength fixes the physical charge density $\rho$ of the boundary theory by $r^{4} E(r) \rightarrow \rho$. The dimensionless magnetic field $\hat{B}=B / \sqrt{\rho}$, temperature $\hat{T}=T / \sqrt{B}$, and entropy density $\hat{s}=s / B^{2}$ are then given by,

$$
\hat{B}=\frac{b}{v_{2} \sqrt{\rho}} \quad \hat{T}=\frac{\sqrt{v_{2}}}{4 \pi \sqrt{b}} \quad \hat{s}=\frac{v_{2}}{4 v_{1} b^{2}}
$$

The equation of state corresponds to the relation $\hat{s}=\hat{s}(k, \hat{T}, \hat{B})$. We shall begin by discussing below analytical solutions available in various limits. Obtaining the function $\hat{s}$ throughout parameter space will, however, require numerical analysis.

\subsection{Flows towards the electric IR fixed point}

In the absence of a magnetic field, $B=0$, the purely electric solution is given by the standard Reissner-Nordstrom form,

$$
U=r^{2}+\frac{q^{2}}{6 r^{6}}-\frac{M}{r^{3}} \quad V_{1}=V_{2}=\ln r \quad E=\frac{\rho}{r^{4}}
$$

In the extremal limit, the location of the horizon $r_{+}$is determined by $U\left(r_{+}\right)=$ $U^{\prime}\left(r_{+}\right)=0$, and the entropy density $s \sim \rho \sim r_{+}^{4}$ does not vanish at $T=0$. The near-horizon geometry of the purely electric solution is $A d S_{2} \times \mathbf{R}^{4}$.

For $B \neq 0$, numerical analysis confirms the existence of a charged magnetic brane solution whose near-horizon behavior coincides with that of the purely electric solution, provided the Chern-Simons coupling $k$ remains below a critical value $k_{c}$ which will be determined shortly. 


\subsection{Flows towards the magnetic IR fixed point}

The near-horizon behavior of the purely magnetic solution is given by $A d S_{4} \times \mathbf{R}^{2}$ space-time at $T=0$, or an $A d S_{4}$ Schwarzschild solution at $T \neq 0$. The two cases may be described together by,

$$
U=\frac{20}{9}\left(r^{2}-\frac{r_{+}^{3}}{r}\right) \quad e^{2 V_{1}}=\frac{20}{9} r^{2} \quad e^{2 V_{2}}=\sqrt{\frac{3}{10}} B
$$

The temperature behaves as $T \sim r_{+}$, and the entropy density may be computed exactly in the low $T$ approximation,

$$
s=\frac{\pi^{2}}{5} \sqrt{\frac{3}{10}} B T^{2}
$$

This $T$-dependence is precisely as expected of a $2+1$-dimensional CFT associated with the near-horizon $A d S_{4}$ space-time.

For $\rho \neq 0$, numerical analysis confirms the existence of a charged magnetic brane solution whose near-horizon behavior is that of the purely magnetic solution, provided $k$ is larger than the critical value $k_{c}$ already identified to end the purely electric flow. The $T^{2}$-dependence of the entropy density which is characteristic of $2+1$ dimensional CFT behavior, persists as long as $k>k_{c}$, but the coefficient is now found to be a non-trivial function,

$$
s=A(k, \hat{B}) B T^{2}
$$

We expect that the problem of calculating the function $A(k, \hat{B})$ may be amenable to analytical treatment, especially since numerical evaluation indicates the following behavior for intermediate and small $\hat{B}$,

$$
A(k, \hat{B}) \sim c(k) \exp \left(d(k) \hat{B}^{-2}\right)
$$

with the characteristic behavior $d(k) \sim\left(k^{2}-k_{c}^{2}\right)^{-1}$ obeyed to remarkable accuracy.

\subsection{Flows towards the Lifshitz IR fixed point}

The near-horizon geometries, $A d S_{2} \times \mathbf{R}^{4}$ for $k<k_{c}$, and $A d S_{4} \times \mathbf{R}^{2}$ for $k>k_{c}$, are separated by a Lifshitz near-horizon geometry at the critical point $k=k_{c}$. Seeking near-horizon solutions which are invariant under space-time scalings, $r \rightarrow \lambda r, t \rightarrow$ $t / \lambda, \quad x_{1,2} \rightarrow \lambda^{-1 / z} x_{1,2}$, and $x_{3,4} \rightarrow \lambda^{-\beta} x_{3,4}$, for real constants $z$ and $\beta$, we find that the existence of such a solution requires $\beta=0$, as well as

$$
k=k_{c}=\frac{1}{\sqrt{3}}
$$


The dynamical scaling exponent $z$ is constrained to the range $z>1$, but otherwise arbitrary. From this scaling behavior in the near-horizon region, the scaling behavior of the low temperature behavior of the entropy density may be deduced, and we find,

$$
\hat{s} \sim T^{\frac{2}{z}}
$$

in accord with the space-dimension of the IR theory being $d=2$, and the parameter $z$ standing for the dynamical critical exponent. As $z$ runs through the range $1<z<\infty$, the entropy density is being interpolated from its IR behavior for the purely electric fixed point at $z=\infty$ to its IR behavior for the purely magnetic fixed point at $z=1$. This interpolating behavior is reflected in the functional dependence of $z$ on the magnetic field $\hat{B}$, which interpolates between the following asymptotic behaviors,

$$
\frac{1}{z}=\left\{\begin{array}{cc}
0.105 \hat{B}^{2} & \hat{B} \ll 1 \\
1-0.894 \hat{B}^{-4} & \hat{B} \gg 1
\end{array}\right.
$$

\subsection{The full phase diagram}

The full phase diagram may now be assembled from the behavior in the various regimes that we have examined in the preceding sections. For large $T$, the entropy density is dominated by temperature alone, and charge density $\rho$ as well as magnetic field $B$ have negligible effects. Thus, we have $s \sim T^{4}$, as expected form the dual field theory by scaling.

For $k<k_{c}$, the flow from $A d S_{6}$ in the UV is towards the Reissner-Nordstrom type near-horizon geometry $A d S_{2} \times \mathbf{R}^{4}$ in the IR with $s \neq 0$ at $T=0$. Without doubt, this holographic solution will become unstable once charged scalar fields, and/or spacedependent modulations are allowed. For $k>k_{c}$, the flow from $A d S_{6}$ in the UV is towards the $A d S_{4} \times \mathbf{R}^{2}$ near-horizon geometry of the purely magnetic brane, with its entropy density behaving as $s \sim B T^{2}$, characteristic of scaling in a 2+1-dimensional CFT. Finally, in the critical region, where $k \sim k_{c}$, the entropy density at low $\hat{T}$ is governed by by a scaling function,

$$
\hat{s}=f\left(k-k_{c}, \hat{B}, \hat{T}\right)
$$

In the absence to date of a (semi-) analytical solution connecting the near-horizon Lifshitz geometry to the asymptotic $A d S_{6}$ region, the scaling function $f\left(k-k_{c}, \hat{B}, \hat{T}\right)$ is accessible only through numerical analysis.

\section{Relation with quantum criticality in condensed matter}

In this section, we shall point towards some exciting, though still speculative, applications of our holographic results to problems in condensed matter physics. One 


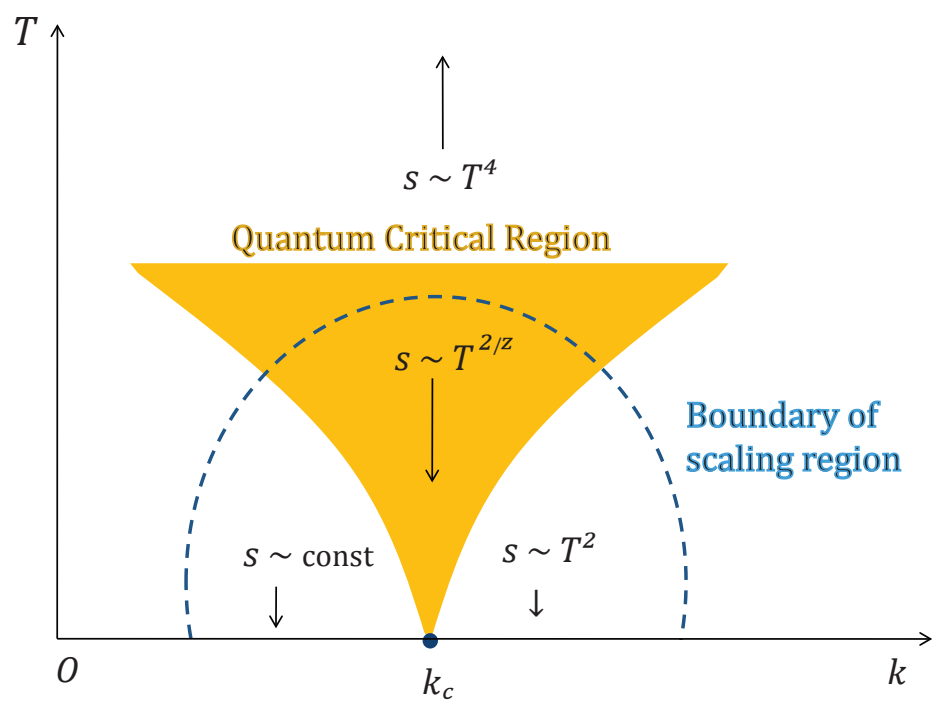

Fig. 6 The full holographic phase diagram in terms of the variables $k$ and $\hat{T}$. To lighten notations, hats on $\hat{T}, \hat{s}$ have not been exhibited in the figure.

application of the 1+1-dimensional quantum criticality problem studied above is to Strontium Ruthenates.

\subsection{Meta-magnetic transitions in Strontium Ruthenates}

The phase transitions exhibited by our holographic systems occur at a finite value of the magnetic field and involve no change of symmetry. These are referred to as meta-magnetic phase transitions. A typical experimental situation is the following. A material has a line of first order phase transitions at finite temperature, reached by dialing the magnetic field, and with the line ending at a finite temperature critical point. By tuning some other control parameter, one can attempt to bring this critical point down to zero temperature, resulting in a quantum critical meta-magnetic transition [52], analogous to what we have found holographically. In particular, thermodynamic quantities such as the specific heat will diverge as the critical magnetic field is approached.

A version of this behavior, with some interesting twists, occurs in the Strontium Ruthenate compound $\mathrm{Sr}_{3} \mathrm{Ru}_{2} \mathrm{O}_{7}$, and has been the subject of much experimental and theoretical interest in the past few years; e.g. [57, 30]. $\mathrm{Sr}_{3} \mathrm{Ru}_{2} \mathrm{O}_{7}$ is a layered material, which, for a large magnetic field perpendicular to the 2-dimensional layers (around $8 \mathrm{~T}$ ) exhibits meta-magnetic behavior with a "shrouded" quantum critical point. Notably, as the magnetic field reaches its critical value the entropy density behaves as $s / T \sim 1 /\left(B-B_{C}\right)$, just as we found in our $\mathrm{AdS}_{5}$ system. There appears to 
be no satisfactory theoretical understanding of this behavior. While this divergence in the entropy density appears to signal the onset of a quantum critical point, what actually seems to happen [54, 30] is that the system evolves into a nematic phase for $7.8 T<B<8.1 T$. Spatial anistropy in the nematic phase can be detected by applying a small in-plane component of magnetic field, which acts to align the domains, and then looking for anistropic behavior of transport coefficients. The nematic phase seems to shroud the quantum critical point in a manner analogous to what occurs in high temperature superconductors. The fact that the would be divergence in $s / T$ is cutoff by the appearance of a nematic phase has been described as nature's solution to the problem of avoiding a non-zero entropy density at zero temperature.

In [57], the complete entropy landscape of $\mathrm{Sr}_{3} \mathrm{Ru}_{2} \mathrm{O}_{7}$ at finite temperature and magnetic field has been mapped out. The parallels with our system are clear, namely the $1 /\left(B-B_{C}\right)$ divergence in the entropy density to temperature ratio; see figure 7 The most obvious difference is that our system is effectively $1+1$ dimensional at the critical point, while $\mathrm{Sr}_{3} \mathrm{Ru}_{2} \mathrm{O}_{7}$ is strongly $2+1$ dimensional. But it is interesting to speculate whether a nematic phase will occur also in our holographic setup. In our ansatz we have assumed full translational and rotational invariance, but recent results indicate that there are frequently instabilities towards anisotropic phases [23, 53, 24, 41, 25, 25, 42].
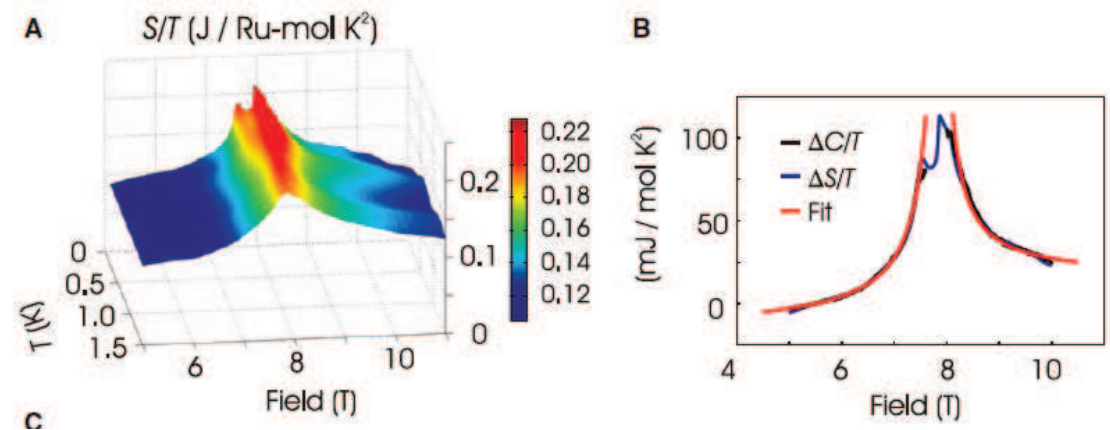

Fig. 7 Entropy landscape of $\mathrm{Sr}_{3} \mathrm{Ru}_{2} \mathrm{O}_{7}$ near the meta-magnetic transition. The right panel illustrates the $1 /\left(B-B_{C}\right)$ divergence of $S / T$, which is ultimately cut off by the appearance of a nematic phase. Figure taken from [57].

\subsection{Relation to Hertz-Millis theory}

A standard approach to modeling magnetically tuned quantum phase transitions is based on the Hertz-Millis theory [39, 51, 49]. In $d+1$ dimensions we consider the effective action 


$$
S=\int d \omega d^{d} k\left(\frac{|\omega|}{|k|}+k^{2}+\left(\hat{B}-\hat{B}_{c}\right)\right)|\phi(\omega, k)|^{2}+\ldots
$$

The bosonic field $\phi$ represents the local magnetization, and one is supposed to think of this action as arising from integrating out gapless fermions at one-loop. There is no controlled approximation that justifies this approach, and indeed it is known to sometimes lead to predictions in conflict with experiment. Let us nonetheless make the following suggestive observations. We consider the action 84 with $d=$ 1 , and compare to our asymptotically $\mathrm{AdS}_{5}$ critical theory. At $\hat{B}=\hat{B_{c}}$ the HertzMillis action is scale invariant, with $k$ and $\omega$ assigned scaling dimensions 1 and 3 respectively. The dynamical critical exponent is therefore $z=3$, which matches our $\mathrm{AdS}_{5}$ result for $k>3 / 4$, and will lead to the scaling law for the entropy, $s \sim$

$T^{1 / 3}$, in one spatial dimension, as we found. Furthermore, $\hat{B}-\hat{B}_{c}$ plays the role of a relevant coupling of scaling dimension 2. This agrees with 62 ; to see this note that the argument of $f$ in $(62)$ has vanishing scale dimension, and $\hat{T}$ shares the same scaling dimension as $\omega$, namely 3 . Therefore, the scaling predictions of the Hertz-Millis theory can be identified in our holographic setup. Of course there are also differences; for instance, there is no analog of our finite ground state entropy density branch for $\hat{B} \leq \hat{B}_{c}$.

\section{Acknowledgments}

This work was supported in part by NSF grant PHY-07-57702.

During the course of this entire project, we have benefited from helpful conversations and correspondence with several colleagues, and we wish to thank here Vijay Balasubramanian, David Berenstein, Sudip Chakravarty, Geoffrey Compère, Jan de Boer, Frédéric Denef, Stéphane Detournay, Tom Faulkner, Jerome Gauntlett, Sean Hartnoll, Gary Horowitz, Finn Larsen, Alex Maloney, Eric Perlmutter, Joe Polchinski, Matt Roberts, Joan Simon, and especially Akhil Shah who collaborated on one of our papers. During parts of this work, we have enjoyed the hospitality of the KITP during the "Quantum Criticality and AdS/CFT Correspondence" program in 2009, and of the Aspen Center for Physics in 2011. One of us (E. D.) wishes to thank the Laboratoire de Physique Théorique de l'Ecole Normale Supérieure, and the Laboratoire de Physqiue Théorique et Hautes Energies, CNRS and Université Pierre et Marie Curie - Paris 6, and especially Constantin Bachas and Jean-Bernard Zuber for their warm hospitality while part of this work was being completed.

\section{References}

1. T. Andrade, J. I. Jottar and R. G. Leigh, "Boundary Conditions and Unitarity: the MaxwellChern-Simons System in $\mathrm{AdS}_{3} / \mathrm{CFT}_{2}$," JHEP 1205, 071 (2012) [arXiv:1111.5054 [hep-th]].

2. O. Aharony, S. S. Gubser, J. M. Maldacena, H. Ooguri and Y. Oz, "Large N field theories, string theory and gravity," Phys. Rept. 323, 183 (2000) [arXiv:hep-th/9905111].

3. A. Almheiri, "Magnetic AdS2 x R2 at Weak and Strong Coupling," arXiv:1112.4820 [hep-th].

4. A. Almuhairi, "AdS 3 and $\mathrm{AdS}_{2}$ Magnetic Brane Solutions," arXiv:1011.1266 [hep-th]. 
5. A. Almuhairi and J. Polchinski, "Magnetic $\mathrm{AdS} \times R^{2}$ : Supersymmetry and stability," arXiv:1108.1213 [hep-th].

6. D. Anninos, G. Compere, S. de Buyl, S. Detournay and M. Guica, "The Curious Case of Null Warped Space," arXiv:1005.4072[hep-th].

7. V. Balasubramanian and P. Kraus, "Space-time and the holographic renormalization group," Phys. Rev. Lett. 83, 3605 (1999) [hep-th/9903190].

8. K. Balasubramanian and J. McGreevy, "Gravity duals for non-relativistic CFTs," Phys. Rev. Lett. 101, 061601 (2008) @arXiv:0804.4053 [hep-th]].

9. V. Balasubramanian and P. Kraus, "A stress tensor for anti-de Sitter gravity," Commun. Math. Phys. 208, 413 (1999) [arXiv:hep-th/9902121].

10. J. de Boer, E. P. Verlinde and H. L. Verlinde, "On the holographic renormalization group," JHEP 0008, 003 (2000) [hep-th/9912012].

11. H. Braviner, R. Gregory and S. F. Ross, "Flows involving Lifshitz solutions," Class. Quant. Grav. 28, 225028 (2011) arXiv:1108.3067[hep-th]].

12. J. D. Brown and M. Henneaux, "Central Charges in the Canonical Realization of Asymptotic Symmetries: An Example from Three-Dimensional Gravity,” Commun. Math. Phys. 104, 207 (1986).

13. M. Cubrovic, J. Zaanen and K. Schalm, "Fermions and the AdS/CFT correspondence: quantum phase transitions and the emergent Fermi-liquid," arXiv:0904.1993 [hep-th].

14. S. de Haro, S. N. Solodukhin and K. Skenderis, "Holographic reconstruction of space-time and renormalization in the AdS / CFT correspondence," Commun. Math. Phys. 217, 595 (2001) |arXiv:hep-th/0002230|.

15. E. D'Hoker, D. Z. Freedman, "Supersymmetric gauge theories and the AdS / CFT correspondence," |hep-th/0201253|.

16. E. D'Hoker and P. Kraus, "Magnetic Brane Solutions in AdS," JHEP 0910, 088 (2009) arXiv:0908.3875 [hep-th]].

17. E. D'Hoker and P. Kraus, "Charged Magnetic Brane Solutions in $\mathrm{AdS}_{5}$ and the fate of the third law of thermodynamics," arXiv:0911.4518 [hep-th].

18. E. D'Hoker and P. Kraus, "Holographic Metamagnetism, Quantum Criticality, and Crossover Behavior," JHEP 1005, 083 (2010) [arXiv:1003.1302 [hep-th]].

19. E. D'Hoker and P. Kraus, "Magnetic Field Induced Quantum Criticality via new Asymptotically $\mathrm{AdS}_{5}$ Solutions," Class. Quant. Grav. 27, 215022 (2010) |arXiv:1006.2573 [hep-th]].

20. E. D'Hoker and P. Kraus, "Charged Magnetic Brane Correlators and Twisted Virasoro Algebras," Phys. Rev. D 84, 065010 (2011) [arXiv:1105.3998 [hep-th]].

21. E. D'Hoker and P. Kraus, "Charge Expulsion from Black Brane Horizons, and Holographic Quantum Criticality in the Plane," arXiv:1202.2085 [hep-th].

22. E. D'Hoker, P. Kraus and A. Shah, "RG Flow of Magnetic Brane Correlators," JHEP 1104, 039 (2011) @arXiv:1012.5072 [hep-th]].

23. S. K. Domokos and J. A. Harvey, "Baryon number-induced Chern-Simons couplings of vector and axial-vector mesons in holographic QCD," Phys. Rev. Lett. 99, 141602 (2007) arXiv:0704.1604 [hep-ph]].

24. A. Donos, J. P. Gauntlett and C. Pantelidou, "Spatially modulated instabilities of magnetic black branes," JHEP 1201, 061 (2012) @arXiv:1109.0471 [hep-th]].

25. A. Donos and J. P. Gauntlett, "Helical superconducting black holes," Phys. Rev. Lett. 108, 211601 (2012) arXiv:1203.0533 [hep-th]].

26. A. Donos, J. P. Gauntlett and C. Pantelidou, "Magnetic and electric AdS solutions in stringand M-theory," arXiv:1112.4195 [hep-th].

27. T. Faulkner, H. Liu, J. McGreevy and D. Vegh, "Emergent quantum criticality, Fermi surfaces, and AdS2," arXiv:0907.2694 [hep-th].

28. T. Faulkner, H. Liu and M. Rangamani, "Integrating out geometry: Holographic Wilsonian RG and the membrane paradigm," arXiv:1010.4036

29. C. Fefferman and C. Robin Graham, "Conformal Invariance", in Elie Cartan et les Mathématiques d'aujourd'hui (Astérisque, 1985) p 95.

30. E. Fradkin, S.A. Kivelson, M. J. Lawler, J. P. Eisenstein, and A. P. Mackenzie, "Nematic Fermi Fluids in Condensed Matter Physics", arXiv:0910.4166 
31. J. P. Gauntlett and O. Varela, "Consistent Kaluza-Klein Reductions for General Supersymmetric AdS Solutions,” Phys. Rev. D 76 (2007) 126007 [arXiv:0707.2315][hep-th]].

32. T. Giamarchi, Quantum Physics in One Dimension, Oxford University Press (2004).

33. K. Goldstein, S. Kachru, S. Prakash and S. P. Trivedi, "Holography of Charged Dilaton Black Holes," arXiv:0911.3586 [hep-th].

34. S. S. Gubser, I. R. Klebanov and A. M. Polyakov, "Gauge theory correlators from non-critical string theory," Phys. Lett. B 428 (1998) 105 [arXiv:hep-th/9802109].

35. S. A. Hartnoll, "Lectures on holographic methods for condensed matter physics," Class. Quant. Grav. 26, 224002 (2009) [arXiv:0903.3246 [hep-th]].

36. S. A. Hartnoll, C. P. Herzog and G. T. Horowitz, "Building a Holographic Superconductor," Phys. Rev. Lett. 101, 031601 (2008) |arXiv:0803.3295[hep-th]].

37. S. A. Hartnoll and L. Huijse, "Fractionalization of holographic Fermi surfaces," arXiv:1111.2606 [hep-th].

38. I. Heemskerk and J. Polchinski, "Holographic and Wilsonian Renormalization Groups," arXiv: 1010.1264

39. J. A. Hertz, "Quantum critical phenomena," Phys. Rev. B 141165 (1976).

40. C. P. Herzog, "Lectures on Holographic Superfluidity and Superconductivity," J. Phys. A A 42, 343001 (2009) arXiv:0904.1975 [hep-th]].

41. N. Iizuka, S. Kachru, N. Kundu, P. Narayan, N. Sircar and S. P. Trivedi, "Bianchi Attractors: A Classification of Extremal Black Brane Geometries," arXiv:1201.4861 [hep-th].

42. N. Iizuka and K. Maeda, "Study of Anisotropic Black Branes in Asymptotically anti-de Sitter," arXiv: 1204.3008 [hep-th].

43. K. Jensen, A. Karch and E. G. Thompson, "A Holographic Quantum Critical Point at Finite Magnetic Field and Finite Density," arXiv:1002.2447 [hep-th].

44. S. Kachru, X. Liu and M. Mulligan, "Gravity Duals of Lifshitz-like Fixed Points," Phys. Rev. D 78, 106005 (2008) |arXiv:0808.1725 [hep-th]].

45. D. E. Kharzeev, L. D. McLerran and H. J. Warringa, "The Effects of topological charge change in heavy ion collisions: 'Event by event P and CP violation',' Nucl. Phys. A 803, 227 (2008) [arXiv:0711.0950 [hep-ph]].

46. I. R. Klebanov, "TASI lectures: Introduction to the AdS / CFT correspondence," hep-th/0009139

47. G. Lifschytz and M. Lippert, "Holographic Magnetic Phase Transition," Phys. Rev. D 80, 066007 (2009) |arXiv:0906.3892 [hep-th]].

48. H. Liu, J. McGreevy and D. Vegh, "Non-Fermi liquids from holography," arXiv:0903.2477 [hep-th].

49. H. v. Lohneysen, A. Rosch, M. Mojta, and P. Wolfle, "Fermi-liquid instabilities at magnetic quantum phase transitions", Rev. Mod. Phys. 79 , 10151075 (2007)

50. J. M. Maldacena, "The large N limit of superconformal field theories and supergravity," Adv. Theor. Math. Phys. 2 (1998) 231 [Int. J. Theor. Phys. 38 (1999) 1113] |arXiv:hep-th/9711200|.

51. A. J. Millis, "Effect of a nonzero temperature on quantum critical points in itinerant fermion systems", Phys. Rev. B 48, 71837196 (1993)

52. A. J. Millis, A. J. Schofield, G. G. Lonzarich and S. A. Grigera, "Metamagnetic quantum criticality in metals," Phys. Rev. Lett. 88, 217204 (2002).

53. S. Nakamura, H. Ooguri and C. S. Park, "Gravity Dual of Spatially Modulated Phase," arXiv:0911.0679 [hep-th].

54. V. Oganesyan, S. A. Kivelson and E. Fradkin, "Quantum theory of a nematic Fermi fuid," Phys. Rev. B 64, 195109 (2001).

55. J. Polchinski, "Introduction to Gauge/Gravity Duality," arXiv:1010.6134 [hep-th].

56. G. Policastro, D. T. Son and A. O. Starinets, "The Shear viscosity of strongly coupled N=4 supersymmetric Yang-Mills plasma," Phys. Rev. Lett. 87, 081601 (2001) |hep-th/0104066].

57. A. W. Rost, R. S. Perry, J.-F. Mercure, A. P. Mackenzie, and S. A. Grigera "Entropy Landscape of Phase Formation Associated with Quantum Criticality in $\mathrm{Sr}_{3} \mathrm{Ru}_{2} \mathrm{O}_{7}$ ", Science Vol. 325. no. 5946, pp. 1360 - 1363 (2009)

58. S. Sachdev, "Quantum Phase Transitions", Cambridge University Press, 2011 
59. N. Seiberg, "Five-dimensional SUSY field theories, nontrivial fixed points and string dynamics," Phys. Lett. B 388, 753 (1996) [hep-th/9608111].

60. D. T. Son, "Toward an AdS/cold atoms correspondence: a geometric realization of the Schroedinger symmetry," Phys. Rev. D 78, 046003 (2008) [arXiv:0804.3972 [hep-th]].

61. N. Ogawa, T. Takayanagi and T. Ugajin, "Holographic Fermi Surfaces and Entanglement Entropy," JHEP 1201, 125 (2012) [arXiv:1111.1023 [hep-th]].

62. E. Witten, "Anti-de Sitter space and holography," Adv. Theor. Math. Phys. 2 (1998) 253 arXiv:hep-th/9802150. 